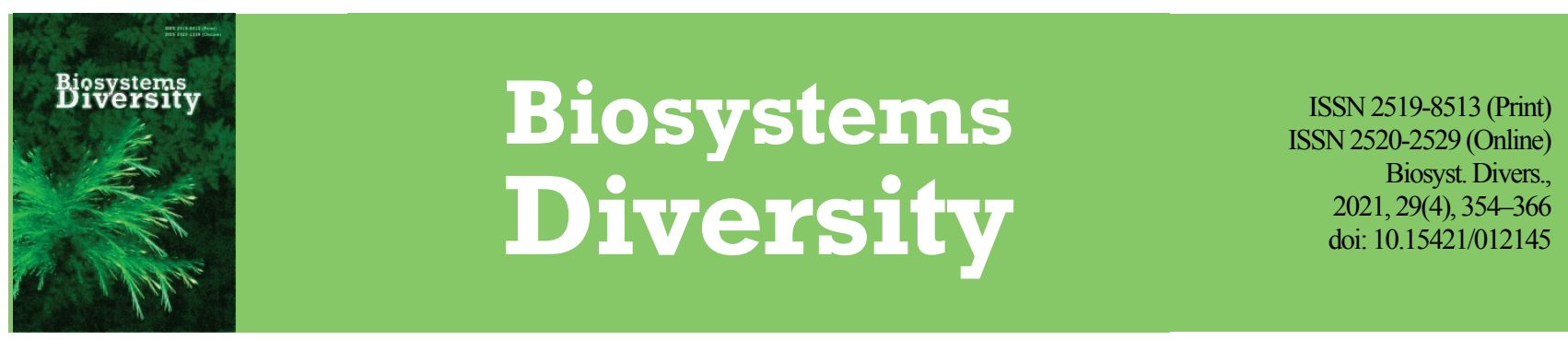

\title{
Synopsis of the order Juncales (Liliopsida) in Ukraine
}

\author{
I. M. Danylyk*, I. G. Olshanskyi** \\ *Institute of Ecology of the Carpathians, Lviv, Ukraine \\ **M. G. Kholodny Institute of Botany, Kyiv, Ukraine
}

Article info

Received 01.10.2021

Received in revised form 25.10.2021

Accepted 27.10.202

Institute of Ecology of the Carpathians,

Kozelnytska st. 4

Lviv, 79026, Ukraine

Tel.: + 38-032-270-74-30.

E-mail:idanylyk@ukr.net

M. G. Kholodny Institute of Botany, Tereshchenkivsk st., 2, Kyiv, 01024, Ukraine Tel.: + 38-044-235-03-74. E-mail:

olshansky1982@ukr.net
Danylyk, I. M., \& Olshanskyi, I. G. (2021). Synopsis of the order Juncales (Liliopsida) in Ukraine. Biosystems Diversity, 29(4), 354 366. doi:10.15421/012145

Juncales is an order of vascular plants with cosmopolitan distribution. Two families of Juncales are present in flora of Ukraine: Juncaceae and Cyperaceae. Through 1985-2021, we conducted comprehensive revision of Juncales in Ukraine. The aim of this research was to compile a checklist of Juncales species in the flora of Ukraine. Our research is based on herbarium materials, literature data and field trips. We collected data in many herbariums of Ukraine and other countries. Our understanding of the taxa was further supplemented by field observations in mountainous (Carpathians and Crimea) and plain territories of Ukraine. Also, we collected information about Juncales species from numerous literature sources. For each taxon, we provided nomenclatural citation and basic synonyms. According to our data, the order Juncales in the flora of Ukraine contains 188 species, belonging to 19 genera. Lists of Juncales species can be found in many thorough publications. For a long time, Checklist of Mosyakin \& Fedoronchuk (1999) was the main list in the nomenclature of vascular plants in Ukraine. To date, many nomenclature and taxonomic changes have been accumulated. For example, we accept genera Schoenoplectiella and Oreojuncus here. Researchers have found many new species for the territory of Ukraine. This information can be found in numerous publications, but is fragmented. Therefore, we have compiled an updated summary of the Juncales species. In Ukrainian territory, Juncales species are considered both widespread and rare. We refer to the rarest species Carex alba, C. bicolor, C. bohemica, C. brunnescens, C. buxbaumii, C. depauperata, C. fuliginosa, C. globularis, C. heleonastes, C. lachenalii, C. loliacea, C. obtusata, C. pediformis, C. rupestris, C. strigosa, Cyperus longus, Eleocharis multicaulis, E. oxylepis, Fimbristylis bisumbellata, Juncus acutiflorus, J. soranthus, J. subnodulosus, Isolepis setacea, Luzula spicata, Schoenoplectus pungens, Trichophorum alpinum, T. cespitosum. Also, the following species are rare: Bolboschoenus yagara, Carex chordorrhiza, C. davalliana, C. dioica, C. hostiana, C. pauciflora, C. secalina, C. vaginata, Cladium mariscus, Eleocharis carniolica, Juncus capitatus, J. castaneus, J. littoralis, J. sphaerocarpus, J. thomasii, J. triglumis, Luzula alpinopilosa, L. sudetica, Schoenoplectiella mucronata, Schoenus ferrugineus.

Keywords: Cyperaceae; Juncaceae; taxonomic diversity; nomenclature; accepted names; synonyms.

\section{Introduction}

In the system of flowering plants (particularly in the class Liliopsida Batsch), the subclass Commelinidae Takht. is marked by a high degree of specialization. Commelinidae is usually divided into several orders (Takhtajan, 2009; Reveal, 2012; Mosyakin, 2013), including the order Juncales Bercht. \& J. Presl. Some authors considered Juncales within the order Poales Small s.l. (APG IV, 2016). However, we consider it appropriate to recognize Juncales as a separate order. The order Juncales is monophyletic and is closely related to Poales. According to Plunkett et al. (1995), Bremer (2002), Jones et al. (2007), Juncales has originated in West Gondwana (South America, and Africa), while Poales has originated in East Gondwana (Australia, Antarctica, India, and Madagascar).

Juncales are perennial, often rhizomatous herbs or annuals, rarely shrubs (Prionium E. Mey.). Stems are round or trigonous, mostly solid. The plants contain no raphides, but silica bodies are present frequently. Leaves are alternate, mostly linear, parallel-veined, rarely reduced or absent; stomata paracytic or sometimes tetracytic. Flowers are small and inconspicuous, solitary or in inflorescences, bisexual or unisexual (monoecious or rarely dioecious). The perianth usually has six segments in two cycles, free, sometimes replaced by bristles or numerous hairs or with no hears. The number of stamens is 3 or 6 . Fruits are nuts, drupe-like, or capsules. Seeds are small. Chromosomes often have diffuse centromere (Takhtajan, 2009; Záveská Drábková, 2013).

Juncales have a cosmopolitan distribution. Juncales species typically grow in damp or cold habitats (Takhtajan, 2009). The order Juncales comprises three families: Thurniaceae Engl., Juncaceae Juss., and Cyperaceae Juss. (Takhtajan, 2009). Some authors rightly recognize Prioniaceae S. L. Munro \& H.P. Linder as a separate family (Munro et al., 2001). Prioniaceae and Thurniaceae are small families, each containing only one small genus (Thurnia Hook. f. and Prionium E. Mey). Thurnia is common in Guyana and the Amazon basin (South America), and Prionium in the Cape Province (South Africa). Juncaceae and Cyperaceae are cosmopolitan. Also, the latter two families are represented in the flora of Ukraine, both with a great taxonomic diversity (Olshanskyi \& Fedoronchuk, 2011, 2012; Danylyk, 2012).

Lists of Juncales species can be found in many detailed publications. For example, they are in Floras, Checklists, and so on. For a long time, Checklist (Mosyakin \& Fedoronchuk, 1999) was the main list in the nomenclature of vascular plants in Ukraine. To date, a large amount of nomenclature has accumulated and many taxonomic changes have been made. Checklists are constantly updated for many groups of plants and for different areas. For example, such lists have recently been published for species of native Italian flora (Bartolucci et al., 2018), ferns and lycophytes of Honduras (Reyes-Chávez et al., 2021), Begonia (Begoniaceae) of Laos (Ding et al., 2020), Myoxanthus (Orchidaceae) (Rojas-Alvarado et al., 2021), etc. In Central and Eastern Europe, Checklists are relatively outdated and need to be updated.

In the botanical taxonomic literature, most Juncales taxa (especially species) are listed under different names (synonyms). We consider it necessary to unify the names of the taxa in accordance with the modern rules of priority. The aim of this research was to compile a checklist of Juncales species in the flora of Ukraine. 


\section{Material and methods}

During 1985-2021, we conducted comprehensive study of Juncales in Ukraine $\left(52^{\circ} 22^{\prime} 46^{\prime \prime}\right.$ and $44^{\circ} 23^{\prime} 11^{\prime \prime}$ parallels of north latitude, $22^{\circ} 08^{\prime} 13^{\prime \prime}$ and $40^{\circ} 13^{\prime} 40^{\prime \prime}$ meridians of east longitude, area of $603,700 \mathrm{~km}^{2}$ ). Therefore, we examined a large number of herbarium specimens in public herbaria relevant for this research (BP, CHER, CWU, DNZ, DSU, KHER, KMF, KRA, KRAM, KW, KWHA, KWHU, LE, LNAU, LW, LWKS, LWS, MELIT, MSK, MSUD, MW, PW, RIVUN, SIMF, UU, YALT). Acronyms are according to Thiers' Index Herbariorum (http://sweetgum.nybg.org/science/ih). We also consulted with some supplementary private herbaria. Our understanding of the taxa was further supplemented by field observations in mountainous (Carpathians and Crimea) and plain territories of Ukraine. Additional information on species was obtained from numerous literature sources. For each taxon we provided the nomenclatural citation and basic synonyms.

The accepted taxonomical names of the order Juncales of Ukraine are based upon the application of comprehensive approach, using modern nomenclatural and taxonomical data on the interpretation of taxa by several authors (the family Cyperaceae: Bolboschoenus (Tatanov, 2004; Hroudova et al., 2007), Carex (Egorova, 1999; Koopman, 2015; Global Carex Group, 2015, 2016), Cyperus (Larridon et al., 2011-2014; Verloove, 2014), Eleocharis (Gonzalez-Elizondo \& Peterson, 1997; Egorova, 2007), Eriophorum (Novoselova, 2001, 2003), Isolepis (Muasya et al., 2000), Schoenoplectiella (Hayasaka, 2012; Shiels et al., 2014; Verloove et al., 2016), Schoenoplectus (Egorova, 2005), Scirpus (Muasya et al., 2001; Egorova, 2004); the family Juncaceae: Juncus (Kirschner et al., 2002b, 2002c), Luzula (Kirschner et al., 2002a); Oreojuncus (Záveská Drábková \& Kirschner, 2013)).

Priority names of taxa and nomenclature combinations are agreed with World Checklist of Selected Plant Families, facilitated by the Royal Botanic Gardens, Kew (http://wcsp.science.kew.org).

\section{Results}

Below are the accepted names taxa of the order Juncales of the flora of Ukraine, and for the species are the most commonly used synonyms in the Ukrainian scientific literature.

\section{Family Cyperaceae Juss}

Blysmus Panz. ex Schult., Mant. 2: 41 (1824), nom. cons.

Blysmus compressus (L.) Panz. ex Link, Hort. Berol. 1: 278 (1827). Schoenus compressus L., Sp. Pl. 1: 43 (1753).

Scirpus planifolius Grimm, Nova Acta Phys.-Med. Acad. Caes. Leop.-Carol. Nat. Cur. 3(App.): 259 (1767).

Scirpus caricis Retz., Fl. Scand. Prod.: 11 (1779), nom. illeg.

Scirpus cariciformis Vest, Man. Bot:: 287 (1805).

Scirpus compressus (L.) Pers., Syn. Pl. 1: 66 (1805), nom. illeg. Blysmus temuis Gilli, Feddes Repert. Spec. Nov. Regni Veg. 46 : 48 (1939).

Scirpus tenuis (Gilli) Parsa, Fl. Iran 5: 435 (1951).

Bolboschoenus (Asch.) Palla, in W. D. J. Koch, Syn. Deut. Schweiz. Fl., ed. 3: 2531 (1905)

Bolboschoenus glaucus (Lam.) S. G. Sm., Novon, 5: 101 (1995).

Scirpus glaucus Lam., Tabl. Encycl. 1: 142 (1791).

Scirpus macrostachys Willd., Enum. Pl.: 78 (1809).

Scirpus maritimus L. var. glaucus (Lam.) Nees R.Wight \& G. A. W. Arnott, Contr. Bot. India: 111 (1834).

Scirpus maritimus L. var. macrostachys Vis., Fl. Dalmat. 1: 109 (1842), nom. illeg.

Bolboschoenus maritimus (L.) Palla var. macrostachys (Willd.) T. V. Egorova, Fl. Severo-Vostoka Evropeiskoi Chasti SSSR 2: 94 (1976).

Bolboschoenus glaucus (Lam.) S. G. Sm. var. macrostachys Tatanov, Novosti Sist. Vyssh. Rast. 39: 69 (2007)
Bolboschoenus laticarpus Marhold, Hroudová, Zákravský \& Ducháček, Phyton (Horn), 44: 7 (2004).

Bolboschoenus maritimus (L.) Palla in W. D. J. Koch, Syn. Deut. Schweiz. Fl., ed. 3: 2532 (1905).

Scirpus maritimus L., Sp. Pl. 1: 51 (1753).

Schoenoplectus maritimus (L.) Lye, Blyttia 29: 145 (1971).

Scirpus compactus Hoffm., Deutsch. Fl. Bot. Taschenb. 3: t. 25 (1800).

Bolboschoenus maritimus (L.) Palla var. compactus (Hoffm.) T. V.Egorova, Fl. Severo-Vostoka Evropeiskoi Chasti SSSR 2: 18 (1976).

Bolboschoenus planiculmis (F. Schmidt) T. V. Egorova, Trudy Bot. Inst. Akad. Nauk S.S.S.R., Ser. 1, Fl. Sist. Vyssh. Rast. 3: 20 (1967). Scirpus planiculmis F. Schmidt, Reis. Amur-Land., Bot.: 190 (1868). Scirpus koshewnikowii Litv., Bull. Soc. Nat. Moscou 58(2): 220 (1882)

Scirpus koshewnikowii Litv., Wild Pl. Tombov Gov.: 142 (1888).

Bolboschoenus koshewnikowii (Litv.) A. E. Kozhevn., Sosud. Rast. Sovet. Dal'nego Vostoka 3: 189 (1988).

Bolboschoenus biconcavus Ohwi, Mem. Coll. Sci. Kyoto Imp. Univ., ser. B, Biol. 18(1): 109 (1944).

Bolboschoenus yagara (Ohwi) Y. C. Yang \& M. Zhan, Acta Biol. Plateau Sin. 7: 14 (1987 publ. 1988).

Bolboschoenus maritimus (L.) Palla var. desoulavii Drobov, Trudy Bot. Muz. Akad. Nauk 11: 91 (1913).

Scirpus yagara Ohwi, Mem. Coll. Sci. Kyoto Imp. Univ., Ser. B., Biol. 18(1): 110 (1944)

Scirpus fluviatilis (Torr.) A.Gray var. yagara (Ohwi) T. Koyama, J. Fac. Sci. Univ. Tokyo, Sect. 3, Bot. 7: 334 (1958).

Bolboschoenus fluviatilis (Torr.) Soják subsp. yagara (Ohwi)

T. Koyama, Acta Phytotax. Geobot. (Kyoto) 31: 140 (1980).

Bolboschoenus yagara (Ohwi) A. E. Kozhevn., Sosud. Rast. Sovet. Dal'nego Vostoka 3: 187 (1988), comb. superfl.

Bolboschoenus desoulavii (Drobov) A. E. Kozhevn., Sosud. Rast. Sovet. Dal'nego Vostoka 3: 188 (1988).

$$
\text { Carex L., Sp. Pl.: } 972 \text { (1753) }
$$

Carex acuta L., Sp. Pl. 2: 978 (1753), excl. var. $\alpha$

Carex gracilis Curtis, Fl. Londin. (Curtis) 4: 282 (1782)

Carex fuscovaginata Kük., Bull. Herb. Boissier, sér. 2, 4: 56 (1903).

Carex graciliformis V. I. Krecz. in P. F. Majevski, Fl. Centr. Russ., ed. 6: 197 (1933)

Carex sareptana V. I. Krecz. in P. F. Majevski, Fl. Centr. Russ., ed. 6 : 197 (1933).

Carex dichroandra V. I. Krecz. in V. L. Komarov (ed.), Fl. URSS 3 : 596 (1935)

Carex acutiformis Ehrh., Beitr. Naturk. 4: 43 (1789). Carex spadicea Roth, Tent. Fl. Germ. 2(2): 461 (1793), nom. illeg. Carex paludosa Gooden., Trans. Linn. Soc. London 2: 202 (1794) Carex olgae Regel, Izv. Imp. Obshch. Lyubit. Estestv. Moskovsk. Univ. 34(2): 83 (1882).

Carex alba Scop. Fl. Carniol., ed. 2, 2: 216 (1772). Carex ajanensis Vorosch., Byull. Glavn. Bot. Sada 60: 35 (1965).

Carex appropinquata Schumach., Enum. Pl. 1: 266 (1801). Carex paradoxa Willd., Schriften Ges. Naturf. Freunde Berlin 1794: 30 (1794), nom. illeg.

Vignea paradoxa Rchb. in J. C. Mössler \& H. G. L. Reichenbach, Handb. Gewächsk. ed. 2, 3: 1621 (1830).

Vignea appropinquata (Schumach.) Soják, Čas. Nár. Mus., Odd. Prír. 148: 194 (1979 publ. 1980).

Carex aterrima Hoppe, Denkschr. Königl.-Baier. Bot. Ges. Regensburg 1:3(1815).

Carex sudetica Opiz, Naturalientausch 11: 413 (1826).

Carex atrata L. subsp. aterrima (Hoppe) Hartm., Sv. Norsk Exc.-Fl.: 131 (1846).

Carex atrata L. subsp. aterrima (Hoppe) Čelak., Prodr. Fl. Böhm.: 67 (1867). 
Carex perfusca V. I. Krecz. in V. L. Komarov (ed.), Fl. URSS 3: 600 (1935).

Carex atrata L. subsp. perfusca (V. I. Krecz.) T. Koyama, J. Jap. Bot. 30: 312 (1955).

Carex cancasica Steven subsp. perfusca (V. I. Krecz.) T. Koyama, J. Fac. Sci. Univ. Tokyo, Sect. 3, Bot. 8(4): 198 (1962).

Carex atherodes Spreng., Syst. Veg. 3: 828 (1826).

Carex aristata R. Br. in J. Franklin, Narr. Journey Polar Sea: 751 (1823), nom. illeg.

Carex orthostachys C. A. Mey. in C. F. von Ledebour, Fl. Altaic. 4: 231 (1833).

Carex siegertiana R. Uechtr., Verh. Bot. Vereins Prov. Brandenburg 8: 103 (1866).

Carex aristata R. Br. subsp. orthostachys (C. A. Mey.) Kük. in H. G. A. Engler (ed.), Pflanzenr., IV, 20(38): 753 (1909).

Carex pergrandis V. I. Krecz. \& Lucznik, Trudy Dal'nevost. Fil. Akad. Nauk S.S.S.R., Ser. Bot. 2: 894 (1937).

Carex atherodes Spreng. var. maxima (Kük.) A. E. Kozhevn., Sosud. Rast. Sovet. Dal'nego Vostoka 3: 342 (1988).

Carex atherodes Spreng. var. orthostachys (C. A. Mey.) A. E. Kozhevn., Sosud. Rast. Sovet. Dal'nego Vostoka 3: 342 (1988). Carex atherodes Spreng. var. vix-vaginata (Kük.) A. E. Kozhevn., Sosud. Rast. Sovet. Dal'nego Vostoka 3: 342 (1988).

Carex atrata L., Sp. Pl.: 976 (1753).

Carex bicolor Bellardi ex All., Fl. Pedem. 2: 267 (1785).

Carex bohemica Schreb., Beschr. Gräs. 2: 52 (1772).

Carex cyperoides L., Syst. Veg. ed. 13: 703 (1774).

Vignea bohemica (Schreb.) Soják, Čas. Nár. Mus. Odd. Přrr. 148: 194 (1979 publ. 1980).

Carex brevicollis DC. in J. B. A. M. de Lamarck \& A. P. de Candolle, Fl. Franç., ed. 3, 5: 295 (1815).

Carex brizoides L., Cent. Pl. I: 31 (1755).

Vignea brizoides (L.) Rchb. in J. C. Mössler \& H. G. L. Reichenbach, Handb. Gewächsk. ed. 2, 3: 1611 (1830).

Carex brunnescens (Pers.) Poir. in J. B. A. M. de Lamarck, Encycl., Suppl. 3: 286 (1813).

Carex curta Gooden. var. brunnescens Pers., Syn. Pl. 2: 539 (1807).

Carex vitilis Fr., Novit. Fl. Suec. Mont., 3: 137 (1842).

Carex canescens L. subsp. brunnescens (Pers.) Asch. \& Graebn., Syn. Mitteleur. Fl. 2(2): 61 (1902).

Carex brunnescens (Pers.) Poir. subsp. vitilis (Fr.) Kalela, Ann. Bot. Fenn. 2: 193 (1965).

Vignea brunnescens (Pers.) Soják, Čas. Nár. Mus., Odd. Prír. 148: 194 (1979 publ. 1980).

Carex buekii Wimm., Jahresber. Schles. Ges. Vaterl. Cult. 29: 83 (1851 publ. 1852).

Carex buxbaumii Wahlenb., Kongl. Vetensk. Acad. Nya Handl. 24: 163 (1803).

Carex polygama Schkuhr, Beschr. Riedgräs. 1: 84 (1801), nom. illeg. Carex polygama Schkuhr subsp. subulata A. Cajand., Ann. Bot. Soc. Zool.-Bot. Fenn. 'Vanamo", 5(5): 11 (1935).

Carex canescens L., Sp. Pl.: 974 (1753), nom. conserv.

Carex cinerea Pollich, Hist. Pl. Palat. 2: 571 (1777).

Carex curta Gooden., Trans. Linn. Soc. London, 2: 145 (1794).

Vignea canescens (L.) Rchb., Fl. Germ. Excurs.: 58 (1830).

Carex hylaea V. I. Krecz. in V. L. Komarov (ed.), Fl. URSS 3: 594 (1935).

Vignea cinerea (Pollichl.) Dostál, Seznam Rostl. Kvét. Českoslov.: 330 (1982).

Carex capillaris L., Sp. Pl.: 977 (1753).

Carex chlorostachys Steven, Mém. Soc. Imp. Naturalistes Moscou 4: 68 (1813).

Carex capillaris L. subsp. chlorostachys (Steven) Á. Löve \& D. Löve \& Raymond, Canad. J. Bot. 35: 749 (1957).

Carex caryophyllea Latourr., Chlor. Lugd.: 27 (1785).

Carex praecox Jacq., Fl. Austr. 5: 23 (1778), nom. illeg.

Carex verna Chaix, Pl. Vapinc.: 8 (1785), nom. illeg.

Carex scabricuspis V. I. Krecz. in V. L. Komarov (ed.), Fl. URSS 3: 609 (1935).
Carex ruthenica V. I. Krecz. in V. L. Komarov (ed.), Fl. URSS 3: 610 (1935).

Carex cespitosa L., Sp. Pl.: 978 (1753).

Vignea neglecta Peterm., Fl. Bienitz: 17 (1841).

Carex cespitosa L. var. retorta Fr., Bot. Not. 1843: 10 (1843).

Carex neglecta (Peterm.) Peterm., Flora, 27: 331 (1844), nom. Illeg.

Carex rubra H. Lévl. \& Vaniot, Bull. Acad. Int. Géogr. Bot., 19: 33 (1909).

Carex inumbrata V. I. Krecz. in V. L. Komarov (ed.), Fl. URSS 3: 218 (1935).

Carex retorta (Fr.) V. I. Krecz. in V. L. Komarov (ed.), Fl. URSS 3: 598 (1935)

Carex chordorrhiza L.f., Suppl.: 414 (1782).

Vignea chordorrhiza (L.f.) Rchb. in J. C. Mössler \& H. G. L. Reichenbach, Handb. Gewächsk. ed. 2, 3: 1608 (1830).

Carex colchica J. Gay, Ann. Sci. Nat., Bot., sér. 2, 10: 303 (1838). Carex ligerica J. Gay, Ann. Sci. Nat., Bot., sér. 2, 10:360 (1838).

Carex schreberi Schrank subsp. ligerica (J. Gay) Almq. in C. J. Hartman, Handb. Skand. Fl., ed. 11: 475 (1879).

Carex colchica J. Gay subsp. ligerica (J. Gay) T. V. Egorova, Novosti Sist. Vyssh. Rast. 10: 104 (1973).

Vignea colchica (J. Gay) Soják, Čas. Nár. Muz., Odd. Prír. 148: 196 (1979 publ. 1980).

Vignea ligerica (J. Gay) Soják, Čas. Nár. Mus., Odd. Prír. 148: 196 (1979 publ. 1980).

Carex curvata Knaf, Flora 30: 184 (1847).

Carex schreberi Schrank subsp. curvata (Knaf) K. Richt., Pl. Eur. 1: 150 (1890).

Carex praecox Schreb. subsp. curvata (Knaf) Kük. in H. G. A. Engler (ed.), Pflanzenr., IV, 20(38): 131 (1909).

Vignea curvata (Knaf) Soják, Cas. Nár. Mus., Odd. Prír. 148: 195 (1979 publ. 1980).

Vignea praecox Schreb. subsp. curvata (Knaf) Dostál, Folia Mus. Rerum Nat. Bohemiae Occid., Bot. 21: 16 (1984).

Carex curvula All., Fl. Pedem. 2: 264 (1785).

Carex dacica Heuff., Flora, 18: 247 (1835).

Carex rigida Gooden., Trans. Linn. Soc. London 2: 193 (1794), nom. illeg.

Vignea dacica (Heuff.) Fuss, Fl. Transsilv.: 681 (1866).

Carex rigida Gooden. var. dacica (Heuff.) Kük. in H. G. A. Engler (ed.), Pflanzenr., IV, 20(38): 302 (1909).

Carex fusca All. subsp. dacica (Heuff.) Serb. \& Nyár. in T. Savulescu, Fl. Republ. Popul. Român. 11: 787 (1966).

Carex bigelowii Torr. ex Schwein. subsp. nardeticola Holub, Folia Geobot. Phytotax. 3(2): 190 (1968).

Carex bigelowii Torr. ex Schwien. subsp. rigida Schultze-Motel, Willdenowia 4(3): 326 (1968).

Carex nigra (L.) Reichard subsp. dacica (Heuff.) Soó, Acta Bot. Acad. Sci. Hung. 16: 370 (1970 publ. 1971).

Carex bigelowii Torr. ex Schwein. subsp. dacica (Heuff.) T. V. Egorova, Fl. Evropeískoí Chasti SSSR 2: 202 (1976).

Carex davalliana Sm., Trans. Linn. Soc. London, 5: 266 (1800).

Carex scabra Hoppe, Bot. Taschernb. Anfänger Wiss. Apothekerkunst 11: 242 (1801).

Vignea davalliana (Sm.) Rchb. in J. C. Mössler \& H. G. L. Reichenbach, Handb. Gewächsk. ed. 2, 3: 1605 (1830).

Carex demissa Hornem., Fors. Oecon. Plantel., ed. 2: 826 (1806).

Carex oederi Retz. subsp. oedocarpa Andersson, Pl. Scand. 1: 25 (1849).

Carex oedocarpa Andersson, Pl. Scand. 1: 26 (1849), nom. superfl.

Carex tumidicarpa Andersson, Bot. Not. 1849: 16(1849).

Carex depauperata Curtis ex Woodw. in W.Withering, Bot. Arr. Brit. Pl. ed. 2, 2: 1049 (1787).

Carex depressa Link, J. Bot. (Schrader) 1799(2): 309 (1800).

Carex depressa subsp. transsilvanica (Schur) K.Richt., Pl. Eur. 1: 158 (1890).

Carex transsilvanica Schur, Verh. Mitth. Siebenbürg. Vereins Naturwiss. Hermannstadt 4: 80 (1853). 
Carex depressa Link var. transsilvanica (Schur) Christ, Bull. Soc. Roy. Bot. Belgique 24(2): 14 (1885).

Carex depressa Link var. euxina Woron. \& Marc., Trudy Imp. S.Peterburgsk. Bot. Sada 24: 564 (1905).

Carex euxina (Woron. \& Marc.) V. I. Krecz. in V. L. Komarov (ed.), Fl. URSS 3: 321 (1935).

Carex depressa Link subsp. transsilvanica (Schur) T. V. Egorova, Novosti Sist. Vyssh. Rast. 9: 80 (1972).

Carex diandra Schrank, Cent. Bot. Anmerk.: 57 (1781).

Carex teretiuscula Gooden., Trans. Linn. Soc. London, 2: 163 (1794).

Vignea teretiuscula (Gooden.) Rchb. in J. C. Mössler \& H. G. L. Reichenbach, Handb. Gewächsk. ed. 2, 3: 1621 (1830).

Vignea diandra (Schrank) Soják, Čas. Nár. Mus., Odd. Prír. 148: 195 (1979 publ. 1980).

Carex digitata L., Sp. Pl.: 975 (1753).

Carex diluta M.Bieb., Fl. Taur.-Caucas. 2: 388 (1808).

Carex axillaris Link, Enum. Hort. Berol. Alt. 2: 329 (1822), nom. illeg.

Carex aitchisonii Boeckeler, Fl. Regensb. 63: 456 (1880).

Carex chorgosica Meinsh., Trudy Imp. S.-Peterburgsk. Bot. Sada 18: 381 (1901).

Carex karelinii Meinsh., Trudy Imp. S.-Peterburgsk. Bot. Sada 18, 3: 380 (1901).

Carex diluta M. Bieb. var. chorgosica (Meinsh.) Kük. in H. G. A. Engler (ed.), Pflanzenr., IV, 20(38): 660 (1909).

Carex diluta M. Bieb. var. karelinii (Meinsh.) Kük. in H. G. A. Engler (ed.), Pflanzenr., IV, 20(38): 660 (1909).

Carex czarwakensis Litv., Trudy Bot. Muz. Imp. Akad. Nauk 7: 97 (1910).

Carex dioica L., Sp. Pl.: 972 (1753).

Vignea dioica (L.) Rchb. in J. C. Mössler \& H. G. L. Reichenbach, Handb. Gewächsk. ed. 2, 3: 1604 (1830).

Carex dioica L. var. isogina Fr., Novit. Fl. Suec. Mant. 3: 135 (1843).

Carex dioica L. subsp. isogyna (Fr.) K.Richt., Pl. Eur. 1: 147 (1890).

Carex distans L., Syst. Nat., ed. 10, 2: 1263 (1759).

Carex disticha Huds., Fl. Angl.: 347 (1762).

Carex intermedia Gooden., Trans. Linn. Soc. London, 2: 154 (1794), nom. illeg.

Vignea disticha (Huds.) Peterm., Pflanzenreich: 150 (1838-1841).

Carex grosheimii V. I. Krecz., in V. L. Komarov (ed.), Fl. URSS 3: 589 (1935).

Carex disticha Huds. subsp. grosheimii (V. I. Krecz.) T. V. Egorova, Novosti Sist. Vyssh. Rast. 22: 54 (1985).

Carex divisa Huds., Fl. Angl.: 348 (1762).

Vignea divisa (Huds.) Rchb., in J. C. Mössler \& H. G. L. Reichenbach, Handb. Gewächsk. ed. 2, 3: 1615 (1830).

Carex coarcta Boott, Proc. Linn. Soc. London 1: 285 (1846).

Carex chaetophylla Steud., Syn. Pl. Glumac. 2: 187 (1855).

Carex divisa Huds. var. chaetophylla (Steud.) Daveau, Bol. Soc. Brot. 9: 100 (1891 publ. 1892).

Carex divulsa Stokes in W.Withering, Bot. Arr. Brit. Pl. ed. 2, 2: 1035 (1787).

Carex guestphalica (Rchb.) Boenn. ex O.Lang, Flora 26: 147 (1843). Vignea divulsa (Stokes) Rchb, in J. C. Mössler \& H. G. L. Reichenbach, Handb. Gewächsk. ed. 2, 3: 1619(1830).

Carex muricata L. subsp. divulsa (Stokes) Celak., Prodr. Fl. Böhmen: 60 (1867).

Carex lumnitzeri (Rouy) V. I. Krecz. in V. L. Komarov (ed.), Fl. URSS 3: 154 (1935), nom. superfl.

Carex echinata Murray, Prodr. Stirp. Gott.: 76 (1770).

Carex stellulata Gooden., Trans. Linn. Soc. London, 2: 144 (1794).

Carex grypos Schkuhr, Beschr. Riedgräs. 2: 18 (1806).

Vignea stellulata (Gooden.) Rchb. in J. C. Mössler \& H. G. L. Reichenbach, Handb. Gewächsk. ed. 2, 3: 1610 (1830).

Vignea echinata (Murray) Fourr., Ann. Soc. Linn. Lyon, n.s., 17: 176 (1869).

Carex elata All., Fl. Pedem. 2: 272 (1785).

Carex elata subsp. elata.
Carex stricta Gooden., Trans. Linn. Soc. London, 2: 196 (1794), nom illeg.

Carex reticulosa Peterm., Flora 27: 333 (1844).

Carex hudsonii A. Benn. in H. C. Watson, London Cat. Brit. Pl., ed. 9: 41 (1895)

Carex elata subsp. omskiana (Meinsh.) Jalas, Ann. Bot. Fenn. 1: 49 (1964).

Carex omskiana Meinsh., Trudy Imp. S.-Peterburgsk. Bot. Sada 18: 340 (1901).

Carex elongata L., Sp. Pl.: 974 (1753).

Vignea elongata (L.) Rchb. in J. C. Mössler \& H. G. L. Reichenbach, Handb. Gewächsk. ed. 2, 3: 1617 (1830).

Carex ericetorum Pollich, Hist. Pl. Palat. 2: 580 (1777).

Carex approximata All., Fl. Pedem. 2: 267 (1785).

Carex extensa Gooden., Trans. Linn. Soc. London, 2: 175 (1794).

Carex flacca Schreb., Spicil. Fl. Lips. App.: 669 (1771).

Carex flacca subsp. flacca

Carex glauca Scop., Fl. Carn., ed. 2, 2: 223 (1772).

Carex flacca subsp. erythrostachys (Hoppe) Holub, Folia Geobot.

Phytotax. 23: 413 (1988).

Carex cuspidata Host, Icon. Descr. Gram. Austriac. 1: 71 (1801).

Carex serrulata Biv., Stirp. Rar. Sicilia. 4: 9 (1818).

Carex erythrostachys Hoppe in J.Sturm, Deutschl. Fl. Abbild. 16: 69 (1835).

Carex glauca Scop. var. erythrostachys (Hoppe) Andersson, Pl. Scand. 1: 31 (1849).

Carex glauca Scop. subsp. serrulata (Biv.) K. Richt., Pl. Eur. 1: 160 (1890).

Carex glauca Scop. var. cuspidata (Host) Asch. \& Graebn., Syn. Mitteleur. Fl. 2(2): 138 (1902), nom. superfl.

Carex flacca Schreb. subsp. cuspidata (Host) Schinz \& R.Keller, Fl. Schweiz, ed. 3, 1: 55 (1914).

Carex flacca Schreb. subsp. serrulata (Biv.) Greuter, Boissiera, 13: 167 (1967).

Carex flava L., Sp. Pl.: 975 (1753).

Carex lepidocarpa Tausch var. laxior Kük., in H. G. A. Engler (ed.), Pflanzenr., IV, 20(38): 673 (1909).

Carex flavella V. I. Krecz. in P. F. Majevski, Fl. Centr. Russ., ed. 6: 185 (1933).

Carex flava L. var. laxior (Kük.) Gleason, Phytologia 4: 22 (1952).

Carex fuliginosa Schkuhr, Beschr. Riedgräs. 1: 91 (1801).

Carex globularis L., Sp. Pl.: 976 (1753).

Carex halleriana Asso, Syn. Stirp. Aragon. 1: 133 (1779).

Carex alpestris All., Fl. Pedem. 2: 270 (1785).

Carex gynobasis Chaix, Pl. Vapinc.: 8 (1785).

Carex hartmaniorum A. Cajander, Ann. Bot. Soc. Zool.-Bot. Fenn. "Vanamo", 5(5): 23 (1935).

Carex buxbaumii Wahlenb. var. australis Andersson, Pl. Scand. 1: 39 (1849).

Carex buxbaumii Wahlenb. var. macrostachya Hartm., Handb. Scand. Fl. ed. 5: 268 (1849).

Carex emasculata V. I. Krecz. in V. L. Komarov (ed.), Fl. URSS 3: 604 (1935).

Carex heleonastes Ehrh. ex L.f., Suppl. Pl.: 414 (1782).

Vignea heleonastes (Ehrh. ex L.f.) Rchb. in J. C. Mössler \& H. G. L. Reichenbach, Handb. Gewächsk. ed. 2, 3: 1609 (1830).

Carex hirta L., Sp. Pl.: 975 (1753).

Carex hordeistichos Vill., Prosp. Hist. Pl. Dauphiné: 18 (1779).

Carex hostiana DC., Cat. Pl. Horti Monsp.: 88 (1813).

Carex fulva Host, Icon. Descr. Gram. Austriac. 4: 53 (1809), nom. illeg.

Carex hornschuchiana Hoppe, Flora (Regensb.) 7: 595 (1824).

Carex fulvescens Mack., Bull. Torrey Bot. Club, 37: 239 (1910).

Carex humilis Leyss., Fl. Halens.: 175 (1761).

Carex buschiorum V. I. Krecz. ex Kolak., Trudy Inst. Abkhaz. Kult. 13:218 (1938).

Carex lachenalii Schkuhr, Beschr. Riedgräs. 1: 51 (1801), nom. cons. Carex tripartita All., Fl. Pedem. 2: 265 (1785), nom. rej.

Carex lagopina Wahlenb., Kongl. Vetensk. Acad. Nya Handl., 24: $145(1803)$ 
Vignea tripartita (All.) Rchb. in J. C. Mössler \& H. G. L. Reichenbach, Handb. Gewächsk. ed. 2, 3: 1609 (1830).

Carex lasiocarpa Ehrh., Hannover. Magaz. 22: 132 (1784). Carex filiformis Gooden., Trans. Linn. Soc. London, 2: 172 (1794).

Carex leersii F. W. Schultz, Flora 53: 459 (1870), nom. cons. Carex cuprina (Sándor ex Heuff.) Nendtv. ex A. Kern., Verh. K. K. Zool.-Bot. Ges. Wien 13: 566 (1863).

Carex muricata L. subsp. leersii (F. W. Schultz) Asch. \& Graebn., Syn. Mitteleur. Fl. 2(2): 40 (1902).

Vignea cuprina (Sándor ex Heuff.) Soják, Cas. Nár. Mus., Odd. Prír. 150: 140 (1981 publ. 1982).

Carex lepidocarpa Tausch, Flora 17: 179 (1834).

Carex flava L. var. elatior Schltdl., Fl. Berol. 1: 477 (1823).

Carex flava L. subsp. brachyrrhyncha Čelak., Prodr. Fl. Bohem. 1: 71 (1867).

Carex viridula Michx. subsp. brachyrrhyncha (Čelak.) B. Schmid var. lepidocarpa (Tausch) B. Schmid, Watsonia, 14: 317 (1983).

Carex viridula Michx. subsp. brachyrrhyncha (Čelak.) B. Schmid var. scotica (E. W. Davies) B. Schmid, Watsonia, 14: 318 (1983).

Carex leporina L., Sp. Pl.: 973 (1753), nom. cons.

Carex ovalis Gooden., Trans. Linn. Soc. London, 2: 148 (1794).

Carex argyroglochin Hornem., Fors. Oecon. Plantel., ed. 3, 1: 927

(1821).

Vignea argyroglochin (Hornem.) Rchb. in J. C. Mössler \& H. G. L. Reichenbach, Handb. Gewächsk. ed. 2, 3: 1612 (1830)

Vignea leporina (L.) Rchb. in J. C. Mössler \& H. G. L. Reichenbach, Handb. Gewächsk. ed. 2, 3: 1613 (1830).

Carex leporina L. var. argyroglochin (Hornem.) W. D. J. Koch, Syn. Fl. Germ. Helv.: 753 (1837).

Vignea ovalis (Gooden.) Soják, Čas. Nár. Mus., Odd. Prír. 148: 196 (1979 publ. 1980).

Carex limosa L., Sp. Pl.: 977 (1753).

Carex fuscocuprea (Kük.) V. I. Krecz. in V. L. Komarov (ed.), Fl. URSS 3: 599 (1935).

Carex liparocarpos Gaudin, Étrennes Fl.: 153 (1804).

Carex liparocarpos subsp. liparocarpos

Carex nitida Host, Icon. Descr. Gram. Austriac. 1: 53, t. 71 (1801).

Carex liparocarpos subsp. bordzilowskii (V. I. Krecz.) T. V. Egorova,

Novosti Sist. Vyssh. Rast. 9: 84 (1972).

Carex schkuhrii Willd., Sp. Pl. ed. 4, 4: 264 (1805).

Carex bordzilowskii V. I. Krecz. in V. L. Komarov (ed.), Fl. URSS 3: 615 (1935).

Carex loliacea L. Sp. Pl.: 974 (1753).

Carex quaternaria Spreng., Syst. Veg. 3: 809 (1826)

Vignea loliacea (L.) Rchb. in J. C. Mössler \& H. G. L. Reichenbach, Handb. Gewächsk. ed. 2, 3: 1610 (1830).

Carex sibirica Willd. ex Kunth, Enum. Pl. 2: 406 (1837).

Carex melanostachya M. Bieb. ex Willd., Sp. Pl. 4, 4: 299 (1805).

Carex nutans Host, Icon. Descr. Gram. Austriac. 1: 61 (1801), nom. illeg.

Carex sulcata Schur, Verh. Mitth. Siebenbürg. Vereins Naturwiss. Hermannstadt 4: 80 (1853).

Carex ledebourii Boiss. \& Buhse, Nouv. Mém. Soc. Imp. Naturalistes Moscou 12: 222 (1860).

Carex ripariiformis Litv., Trudy Bot. Muz. Imp. Akad. Nauk 7: 96 (1910).

Carex michelii Host, Syn. Pl. Austr.: 507 (1797).

Carex montana L., Sp. Pl.: 975 (1753).

Carex muricata L., Sp. Pl.: 974 (1753).

Carex muricata subsp. muricata

Vignea muricata (L.) Rchb. in J. C. Mössler \& H. G. L. Reichenbach, Handb. Gewächsk. ed. 2, 3: 1611 (1830).

Carex muricata subsp. ashokae A. M. Molina, Acedo \& Llamas, Syst. Bot. 33: 248 (2008).

Carex nigra (L.) Reichard, Fl. Moeno-Francof. 2: 96 (1778).

Carex nigra subsp. nigra

Carex acuta L. var. nigra L., Sp. Pl.: 978 (1753).

Carex fusca All., Fl. Pedem. 2: 269 (1785).
Carex goodenoughii J. Gay, Ann. Sci. Nat. Bot., sér. 2, 11: 191 (1839).

Carex vulgaris Fries, Novit. Fl. Suec. Mant. 3: 153 (1842), nom. superfl.

Carex nigra subsp. juncea (Fr.) Soó, Feddes Repert. 83: 148 (1972).

Carex vulgaris Fr. subsp. juncea Fr., Novit. Fl. Suec. Mant. 3: 154 (1842)

Carex juncella (Fr.) Th. Fr., Bot. Not. 1857: 207 (1857).

Carex juncella subsp. wiluica (Meinsh. ex Maack) T. V. Egorova, Novosti Sist. Vyssh. Rast. 9: 92 (1972).

Carex nigra subsp. wiluica (Meinsh. ex Maack) Á. Löve \& D. Löve, Bot. Not. 128: 505 (1975 publ. 1976).

Carex obtusata Lilj., Kuhgl. Vetensk. Akad. Nya Handl. 14: 69 (1793). Carex spicata Shkuhr, Beschr. Abbild. Riedgras. 1: 11 (1801), nom. illeg.

Carex microcephala C. A. Mey. in C. F. von Ledebour, Fl. Altaic. 2: 205 (1830).

Carex oederi Retz., Fl. Scand. Prodr.: 179 (1779).

Carex viridula Michx., Fl. Bor.-Amer. 2: 170 (1803).

Carex serotina Mérat, Nouv. Fl. Env. Paris, ed. 2, 2: 54 (1821).

Carex oederi Retz. var. viridula (Michx.) Kük. in H. G. A. Engler (ed.), Pflanzenr., IV, 20(38): 674 (1909).

Carex ornithopoda Willd., Sp. Pl. 4, 4: 255 (1805).

Carex pedata L., Sp. Pl. ed. 2: 1384 (1763), nom. rej.

Carex pedata All., Fl. Pedem. 2: 168 (1785), nom. illeg.

Carex digitata L. var. ornithopoda (Willd.) DC. in J. B. A. M. de Lamarck \& A. P. de Candolle, Fl. Franç., ed. 3, 5: 291 (1815).

Carex digitata L. subsp. ornithopoda (Willd.) Bonnier \& Layens, Tabl. Syn. Pl. Vasc. France: 334 (1894).

Carex otrubae Podp., Spisy Prír. Fak. Masarykovy Univ. 12: 15 (1922). Carex nemorosa Rebent., Prodr. Fl. Neomarch.: 21 (1804), sensu auct.

Carex vulpina L. var. nemorosa DC. in J. B. A. M. de Lamarck \& A. P. de Candolle, Fl. Franç., ed. 3, 5: 288 (1815).

Carex vulpina L. var. subcontigua Kük. in H. G. A. Engler (ed.), Pflanzenr., IV, 20(38): 170 (1909).

Vignea otrubae (Podp.) Soják, Cas. Nár. Mus., Odd. Prír. 148: 196 (1979 publ. 1980).

Carex cuprina (Sándor ex Heuff.) Nendtv. ex A. Kern. var. subcontigua (Kük.) De Langhe \& Lambinon, Nouv. Fl. Belg., Grand-Duché Luxemb., Nord France, ed. 3: 922 (1983).

Carex pairae F. W. Schultz, Flora 51: 303 (1868).

Carex loliacea Schkuhr, Beschr. Riedgräs. 1: 22 (1801), nom. illeg.

Carex muricata L. subsp. pairae (F. W. Schultz) Celak., Kvet. Okolí Praz.: 43 (1870).

Carex panicea L., Sp. P1.: 977 (1753).

Carex paniculata L., Cent. Pl. 1:32 (1755).

Carex paniculata subs. paniculata

Vignea paniculata (L.) Rchb. in J. C. Mössler \& H. G. L. Reichenbach, Handb. Gewächsk. ed. 2, 3: 1622 (1830).

Carex paniculata subsp. szovitsii (V. I. Krecz.) Ö. Nilsson in P. H. Davis (ed.), Fl. Turkey, 9: 91 (1985).

Carex pauciflora Lightf., Fl. Scot. 2: 543 (1777).

Carex pediformis C. A. Mey., Mém. Acad. Imp. Sci. St.-Pétersbourg Divers Savans 1: 219 (1831).

Carex kirilowii Turcz., Bull. Soc. Naturalistes Moscou, 28(I): 340 (1855).

Carex pediformis C. A. Mey. var. floribunda Korsh., Trudy Imp. S.Peterburgsk. Bot. Sada 12: 409 (1892).

Carex floribunda (Korsh.) Meinsh., Trudy Imp. S.-Peterburgsk. Bot. Sada 18: 402 (1901), nom. illeg.

Carex sutschanensis Kom., Izv. Imp. Bot. Sada Petra Velikago 16: 155 (1916).

Carex pediformis C. A. Mey. f. leopolitana Kük. in H. G. A. Engler (ed.), Pflanzenr., IV, 20(38): 491 (1909).

Carex hankaensis Kitag., J. Jap. Bot. 17: 236 (1941).

Carex woroschilovii A. E. Kozhevn., Byull. Moskovsk. Obshch. Isp.

Prir., Otd. Biol., n.s., 92(6): 128 (1987).

Carex pendula Huds., Fl. Angl.: 352 (1762). 
Carex maxima Scop., Fl. Carniol., ed. 2, 2: 229 (1772)

Carex pilosa Scop., Fl. Carniol., ed. 2, 2: 226 (1772).

Carex pilulifera L., Sp. Pl.: 976(1753).

Carex praecox Schreb., Spic. Fl. Lips.: 63 (1771).

Carex schreberi Schrank, Baier. Fl., 1: 278 (1789).

Vignea praecox (Schreb.) Soják, Cas. Nár. Mus., Odd. Prír. 148: 197 (1979 publ. 1980).

Carex pseudocyperus L., Sp. Pl.: 978 (1753).

Carex remota L., Fl. Angl.: 24 (1754).

Vignea remota (L.) Rchb. in J. C. Mössler \& H. G. L. Reichenbach, Handb. Gewächsk. ed. 2, 3: 1616(1830).

Carex rhizina Blytt ex Lindblom, Bot. Not. 1839(8): 98 (1839). Carex rhizodes Blytt ex Boott, Ill. Gen. Carex 4: 196 (1867). Carex pediformis C. A. Mey. subsp. rhizodes (Blytt ex Boott) H. Lindb., Exsicc. (Pl. Finland.) 1-8: 44 (1907).

Carex pediformis C. A. Mey. var. rhizina (Blytt ex Lindblom) Kük in H. G. A. Engler (ed.), Pflanzenr., IV, 20(38): 491 (1909).

Carex pediformis C. A. Mey. subsp. rhizina (Blytt ex Lindblom) Printz, Veg. Siber.-Mongol. Front.: 151 (1921).

Carex riparia Curtis, Fl. Londin. 4: t. 60 (1783)

Carex rostrata Stokes, in W. Withering, Bot. Arr. Brit. Pl. ed. 2, 2: 1059 (1787), nom. cons.

Carex inflata Huds., Fl. Angl:: 354 (1762), nom. rej.

Carex ampullacea Gooden., Trans. Linn. Soc. London 2: 207 (1794), nom. superfl.

Carex rupestris All., Fl. Pedem. 2: 264 (1785).

Carex petraea Wahlenb., Kongl. Vetensk. Acad. Nya Handl. 24: 139 (1803).

Carex secalina Willd. ex Wahlenb., Kongl. Vetensk. Acad. Nya Handl.

24: 151 (1803).

Carex hordeistichos Vill. var. microstachys Schur, Enum. Pl. Transsilv:: 710 (1866).

Carex sempervirens Vill., Hist. Pl. Dauphiné. 2: 214 (1787).

Carex sempervirens Vill. var. pseudotristis Domin, Rozpr. Česke Akad. Ved, Tr. 2, Vedy Mat. Prír. 41(9): 8 (1931).

Carex sempervirens Vill. subsp. pseudotristis (Domin) Pawł., Publ. Inst. Bot. Univ. Jagell. Cracov. 1937: 5 (1937).

Carex spicata Huds., Fl. Angl:: 349 (1762).

Carex contigua Hoppe in J. Sturm, Deutschl. Fl. Abbild. 14: 61 (1833).

Carex muricata L. var. contigua (Hoppe) Fiori, Nuov. Fl. Italia 1: 185 (1923).

Vignea spicata (Huds.) Soják, Cas. Nár. Mus., Odd. Prír. 148: 197 (1979 publ. 1980).

Carex stenophylla Wahlenb., Kongl. Vetensk. Acad. Nya Handl. 24: 142 (1803).

Carex juncifolia Host, Syn. Pl.: 504 (1797), nom. illeg.

Carex glomerata Host, Icon. Descr. Gram. Austriac. 1: 34, t. 44 (1801), nom. illeg.

Carex hostii Schkuhr, Beschr. Riedgräs. 2: 7 (1806).

Vignea stenophylla (Wahlenb.) Rchb., Fl. Germ. Excurs.: 56 (1830).

Carex uralensis C. B. Clarke, Bull. Misc. Inform. Kew, Addit. Ser. 8: 76 (1908).

Carex strigosa Huds., Fl. Angl., ed. 2: 411 (1778).

Carex supina Willd. ex Wahlenb., Kongl. Vetensk. Acad. Nya Handl. 24: 158 (1803)

Carex verna Schkuhr, Beschr. Riedgräs. 1: 89 (1801), nom. illeg. Carex conglobata Kit. ex Willd., Sp. Pl., ed. 4, 4: 281 (1805), nom. illeg.

Carex sphaerocarpa Willd., Sp. Pl., ed. 4, 4: 265 (1805), nom. illeg.

Carex obtusata Lilj. var. spicata Asch., Fl. Brandenburg 1: 779 (1864).

Carex sylvatica Huds., Fl. Angl:: 353 (1762).

Carex patula Scop., Fl. Carniol., ed. 2, 2: 226 (1772).

Carex drymeia L.f., Suppl. Pl.: 414 (1782).

Carex tomentosa L., Mant. Pl. 1: 123 (1767).

Carex subvillosa M. Bieb., Fl. Taur.-Caucas. 2: 386(1808).

Carex umbrosa Host, Icon. Descr. Gram. Austriac. 1: 52 (1801).

Carex longifolia Host, Icon. Descr. Gram. Austriac. 4: 48, t. 85 (1809), nom. illeg.
Carex praecox Schreb. var. longifolia Wahlenb., Fl. Carpat. Princ.: 300 (1814)

Carex polyrrhiza Wallr., Sched. Crit.: 492 (1822).

Carex umbrosa Host var. longifolia (Wahlenb.) Nyman, Consp. Fl. Eur.: 773 (1882)

Carex vaginata Tausch, Flora 4: 557 (1821).

Carex panicea L. var. sparsiflora Wahlenb., Fl. Lapp.: 236(1812).

Carex sparsiflora (Wahlenb.) Steud., Nomencl. Bot., ed. 2, 1: 296 (1840)

Carex vaginata Tausch var. gruetteri Asch. \& Graebn., Syn. Mitteleur. Fl. 2(2): 144 (1902).

Carex sparsiflora Wahlenb. var. gruetteri (Asch. \& Graebn.) Kük. in H. G. A. Engler (ed.), Pflanzenr., IV, 20(38): 513 (1909).

Carex vesicaria L., Sp. Pl.: 979 (1753)

Carex vulpina L., Sp. Pl.: 973 (1753).

Carex compacta Lam., Fl. Franç., ed. 2, 2: 172 (1779), nom. superfl. Carex spicata Thuill., Fl. Env. Paris, ed. 2: 480 (1790), nom. illeg.

Vignea vulpina (L.) Rchb. in J. C. Mössler \& H. G. L. Reichenbach, Handb. Gewächsk. ed. 2, 3: 1620 (1830).

Carex vulpina L. var. compacta (Lam.) Velen., Fl. Bulg.: 576(1891).

Carex glomerata Gilib. ex Bubani, Fl. Pyren. 3: 221 (1902), nom. illeg.

Cladium P. Browne, Civ. Nat. Hist. Jamaica: 114 (1756).

Cladium mariscus (L.) Pohl, Tent. Fl. Bohem. 1: 32 (1809).

Schoenus mariscus L., Sp. Pl. 1: 42 (1753).

Isolepis martii Roem. \& Schult., Syst. Veg., ed. 15, bis 2: 117 (1817).

Cladium martii (Roem. \& Schult.) K.Richt., Pl. Eur. 1: 144 (1890).

Cladium mariscus (L.) Pohl var. martii (Roem. \& Schult.) Kük., Repert. Spec. Nov. Regni Veg. 31: 185 (1942).

Cladium grossheimii Pobed. Bot. Mater. Gerb. Bot. Inst. Komarova Akad. Nauk S.S.S.R. 12: 13 (1950).

Cladium mariscus (L.) Pohl subsp. martii (Roem. \& Schult.) T. V. Egorova, Fl. Evropeiskoi Chasti SSSR 2: 129(1976).

$$
\text { Cyperus L., Sp. Pl.: } 44 \text { (1753). }
$$

Cyperus difformis L., Cent. Pl. II: 6(1756)

*Cyperus esculentus L., Sp. Pl.: 45 (1753).

Cyperus aureus Ten., Fl. Napol. 3: 45 (1824).

Pterocyperus esculentus (L.) Opiz, Seznam: 80 (1852)

Cyperus aureus Ten. subsp. esculentus (L.) Nyman, Consp. Fl. Eur.: 759 (1882), nom. superfl.

Cyperus flavescens L., Sp. Pl.: 46 (1753).

Pycreus flavescens (L.) P. Beauv. ex Rchb., Fl. Germ. Excurs.: 72 (1830).

Cyperus fuscus L., Sp. Pl.: 46 (1753)

Cyperus glaber L., Mant. Pl. 2: 179 (1771).

Chlorocyperus glaber (L.) Palla, Alllg. Bot. Z. Syst. 6: 201 (1901).

Dichostylis glabra (Palla) Palla, Vestn. Tiflissk. Bot. Sada 21: 24 (1912)

Pycreus glaber (L.) Hayek, Repert. Spec. Nov. Regni Veg. Beih. 30(3): 146 (1932).

Cyperus glomeratus L., Cent. Pl. II: 5 (1756).

Chlorocyperus glomeratus (L.) Palla, Allg. Bot. Z. Syst. 6: 61 (1900). Pycreus glomeratus (L.) Hayek, Repert. Spec. Nov. Regni Veg. Beih. 30(3): 145 (1932).

Cyperus hamulosus M. Bieb., Fl. Taur.-Caucas. 1: 35 (1808).

Scirpus hamulosus (M. Bieb.) Steven, Mém. Soc. Imp. Naturalistes Moscou 4: 356 (1814).

Isolepis hamulosa (M. Bieb.) Kunth, Enum. Pl. 2: 204 (1837).

Dichostylis hamulosa (M. Bieb.) Nees, Linnaea 9: 289 (1834).

Mariscus hamulosus (M. Bieb.) S. S. Hooper, Kew Bull. 26: 578 (1972).

Cyperus longus L., Sp. Pl.: 45 (1753).

Cyperus longus subsp. longus

Chlorocyperus longus (L.) Palla, Allg. Bot. Z. Syst. 6: 61 (1900)

Cyperus longus subsp. badius (Desf.) Bonnier \& Layens, Tabl. Syn. Pl.

Vasc. France: 324 (1894). 
Cyperus badius Desf., Fl. Atlant. 1: 45 (1798).

Chlorocyperus badius (Desf.) Palla, Allg. Bot. Z. Syst 6: 61 (1900).

Cyperus michelianus (L.) Delile, Descr. Egypte, Hist. Nat. 3: 50 (1813).

Scirpus michelianus L., Sp. Pl.: 52 (1753).

Cyperus michelianus (L.) Link, Hort. Berol. 1: 303 (1827), comb. superfl.

Dichostylis micheliana (L.) Nees in R. Wight, Contr. Bot. India: 94 (1834).

Dichostylis wolgensis A. O. Tarassov, Pochvy Rast. Yugo-Vostoka 1970: 93 (1970).

Cyperus odoratus L., Sp. Pl.: 46(1753).

Cyperus ferax Rich., Actes Soc. Hist. Nat. Paris 1: 106(1792)

Torulinium ferax (Rich.) Ham., Prodr. Pl. Ind. Occid.: 15 (1825).

Torulinium odoratum (L.) S. S. Hooper, Kew Bull. 26: 579 (1972).

Cyperus pannonicus Jacq., Fl. Austriac. 5: 29 (1778).

Pycreus pannonicus (Jacq.) P. Beauv. ex Rchb., Fl. Germ. Excurs.: 72 (1830).

Chlorocyperus pannonicus (Jacq.) Rikli, Jahrb. Wiss. Bot. 27: 563 (1895).

Acorellus pannonicus (Jacq.) Palla in W. D. J. Koch, Syn. Deut. Schweiz. Fl., ed. 3, 2: 2557 (1905).

Juncellus pannonicus (Jacq.) C. B. Clarke, Bull. Misc. Inform. Kew, Addit. Ser. 8: 3 (1908).

Cyperus serotinus Rottb., Descr. Icon. Rar. Pl.: 31; Prog.: 18 (1773).

Juncellus serotinus (Rottb.) C. B. Clarke in J. D. Hooker, Fl. Brit. India 6: 594 (1893).

Chlorocyperus serotinus (Rottb.) Palla, Allg. Bot. Z. Syst. 6: 221 (1900).

Duval-jouvea serotina (Rottb.) Palla, in W. D. J. Koch, Syn. Deut. Schweiz. Fl., ed. 3, 3: 2556 (1905)

Eleocharis R. Br., Prodr. Fl. Nov. Holland.: 224 (1810).

Eleocharis acicularis (L.) Roem. \& Schult., Syst. Veg., ed. 15 bis 2: 154 (1817).

Scirpus acicularis L., Sp. Pl.: 48 (1753).

Eleocharis carniolica W. D. J. Koch, Syn. Fl. Germ. Helv., ed. 2: 853 (1844).

Eleocharis mamillata (H. Lindb.) H. Lindb., Exsicc. (Herb. Norm.) 44 108 (1902)

Eleocharis mamillata subsp. mamillata

Scirpus mamillatus H. Lindb., Acta Soc. Fauna Fl. Fenn. 23(7): 7 (1902).

Eleocharis mamillata subsp. austriaca (Hayek) Strandh., Opera Bot 9(2): 9 (1965)

Eleocharis austriaca Hayek, Sched. Fl. Stiriac. 19-20: 8 (1910).

Eleocharis leptostylopodiata Zinserl. in V. L. Komarov (ed.), Fl. URSS 3: 581 (1935).

Eleocharis mitracarpa Steud., Syn. Pl. Glumac. 2: 77 (1854).

Scirpus equisetiformis Meinsh., Trudy Imp. S.-Peterburgsk. Bot. Sada 18: 261 (1901).

Eleocharis palustris (L.) Roem. \& Schult. subsp. austriaca (Hayek) Podp., Práce Morav. Prír. Spolecn. 5: 50 (1929).

Eleocharis argyrolepidoides Zinserl. in V. L. Komarov (ed.), Fl. URSS 3: 584 (1935).

Eleocharis multicaulis (Sm.) Desv., Observ. Pl. Angers: 74 (1818). Scirpus multicaulis Sm., Fl. Brit. 1: 48 (1800).

Eleocharis ovata (Roth) Roem. \& Schult., Syst. Veg., ed. 15 bis 2: 152 (1817).

Scirpus ovatus Roth, Tent. Fl. Germ. 2(2): 562 (1793).

Scirpus soloniensis Dubois, Méth. Éprouv., ed. 2: 249 (1833).

Eleocharis soloniensis (Dubois) H. Hara, J. Jap. Bot. 14: 338 (1938).

Eleocharis oxylepis (Meinsh.) B. Fedtsch., Rastitel'n. Turkestana: 165 (1915).

Scirpus oxylepis Meinsh., Trudy Imp. S.-Peterburgsk. Bot. Sada 18: 263 (1901).

Eleocharis palustris (L.) Roem. \& Schult., Syst. Veg., ed. 15 bis 2: 151 (1817).

Scirpus palustris L., Sp. Pl.: 47 (1753).
Eleocharis eupalustris H. Lindb., Acta Soc. Fauna Fl. Fenn. 27(7): 5 (1902)

Eleocharis intersita Zinserl. in V. L. Komarov (ed.), Fl. URSS 3: 581 (1935).

Eleocharis crassa Fisch. \& C. A. Mey. ex Zinserl. in V. L. Komarov (ed.), Fl. URSS 3: 582 (1935).

Eleocharis levinae Zoz, Fl. RSS Ucr. 2: 426 (1940).

Eleocharis oxystachys Sakalo, Novosti Sist. Vyssh. Nizsh. Rast. 1976: 50 (1977).

Eleocharis parvula (Roem. \& Schult.) Link ex Bluff, Nees \& Schauer, Comp. Fl. German., ed. 2, 1: 93 (1836).

Scirpus parvulus Roem. \& Schult., Syst. Veg., ed. 15 bis 2: 124 (1817).

Eleocharis parvula (Roem. \& Schult.) Link ex Bluff, Nees \& Schauer subsp. oppermannii Zoz, Trudy Inst. Bot. Kharkivs'k. Derzhavn. Univ. 1: 46 (1936).

Eleocharis quinqueflora (Hartmann) O. Schwarz, Mitt. Thüring. Bot. Ges. 1: 89 (1849).

Scirpus quinqueflorus Hartmann, Primae Lin. Inst. Bot., ed. 2: 85 (1767).

Scirpus pauciflorus Lightf., Fl. Scot. 2: 1078 (1777).

Eleocharis pauciflora (Lightf.) Link, Hort. Berol. 1: 284 (1827).

Eleocharis meridionalis Zinserl. in V.L.Komarov (ed.), Fl. URSS 3: 580 (1935).

Eleocharis czernjajevii Zoz, Trudy Inst. Bot. Kharkivs'k. Derzhavn. Univ. 1: 55 (1936)

Eleocharis quinqueflora (Hartmann) O. Schwarz subsp. meridionalis (Zinserl.) T. V. Egorova, Novosti Sist. Vyssh. Rast. 18: 102 (1981).

Eleocharis uniglumis (Link) Schult., Mant. 2: 88 (1824).

Scirpus uniglumis Link, Jahrb. Gewächsk. 1(3): 77 (1820).

Scirpus klingei Meinsh., Trudy Imp. S.-Peterburgsk. Bot. Sada 18. 262 (1901).

Eleocharis klingei (Meinsh.) B. Fedtsch., Rastitel'n. Turkestana: 165 (1915).

Eleocharis korshinskyana Zinserl., Trudy Glavn. Bot. Sada 40: 280 (1929).

Eleocharis scythica Zinserl., Trudy Glavn. Bot. Sada 40: 279 (1929).

Eleocharis euniglumis Zinserl. in V. L. Komarov (ed.), Fl. URSS 3: 584 (1935).

Eleocharis multiseta Zinserl. in V. L. Komarov (ed.), Fl. URSS 3 : 586 (1935).

Eleocharis paucidentata Zinserl. in V. L. Komarov (ed.), Fl. URSS 3: 588 (1935).

Eleocharis macrocarpa Zoz, Trudy Inst. Bot. Kharkivs'k. Derzhavn Univ. 1: 55 (1936).

Eleocharis zinszerlingii Zoz, Trudy Inst. Bot. Kharkivs'k. Derzhavn. Univ. 1: 55 (1936).

Eleocharis carinata Sakalo, Novosti Sist. Vyssh. Nizsh. Rast. 1976: 48 (1977).

Eriophorum L., Sp. Pl. 1: 52 (1753).

Eriophorum angustifolium Honck., Verz. Gew. Teutschl. 1: 153 (1782).

Eriophorum polystachion L., Sp. Pl.: 52 (1753), nom. rej.

Eriophorum gracile W. D. J. Koch in A. W. Roth, Catal. Bot. 2(Add.): 259 (1799).

Eriophorum coreanum Palla, Österr. Bot. Z. 59: 190 (1909).

Eriophorum latifolium Hoppe, Bot. Taschenb. Anfänger Wiss. Apothekerkunst 11: 108(1801).

Eriophorum vaginatum L. Sp. Pl.: 52 (1753).

Fimbristylis Vahl, Enum. Pl. Obs. 2: 285 (1805), nom. cons.

Fimbristylis bisumbellata (Forssk.) Bubani, Dodecanthea: 30 (1850) Scirpus bisumbellatus Forssk. Fl. Aegypt.-Arab.: 15 (1775). Fimbristylis dichotoma (L.) Vahl subsp. bisumbellata (Forssk.) Luceño, Anales Jard. Bot. Madrid 57: 176(1999). 
Isolepis setacea (L.) R. Br., Prodr. Fl. Nov. Holland.: 222 (1810). Scirpus setaceus L., Sp. Pl.: 49 (1753).

Mariscus setaceus (L.) Moench, Methodus: 350 (1794).

Cyperus setaceus (L.) Missbach \& E. H. L. Krause in J. Sturm, Deutschl. Fl. Abbild., ed. 2, 2: 21 (1900), nom. illeg.

Schoenoplectus setaceus (L.) Palla, in W. D. J. Koch, Syn. Deut. Schweiz. Fl., ed. 3: 2538 (1905).

Rhynchospora Vahl, Enum. Pl. Obs. 2: 229 (1805), nom. cons.

Rhynchospora alba (L.) Vahl, Enum. Pl. Obs. 2: 236 (1805). Schoenus albus L., Sp. Pl.: 44 (1753).

\section{Schoenoplectiella Lye, Lidia 6: 20 (2003)}

Schoenoplectiella melanosperma (C. A. Mey.) Danylyk, Olshanskyi \& Zhygalova, Phytotaxa 299(1): 138 (2017).

Scirpus melanospermus C. A. Mey., Acad. Imp. Sci. St.-Pétersbourg Divers Savans 1: 199 (1831).

Scirpus supinus L. var. melanospermus (C. A. Mey.) Schmalh., Fl. Sredn. Yuzhn. Rossii 2: 545 (1897).

Schoenoplectus melanospermus (C. A. Mey.) Grossh., Fl. Kavkaza 1: 146 (1928)

Schoenoplectiella mucronata (L.) J. Jung \& H. K. Choi, J. Pl. Biol. 53(3): 230 (2010).

Scirpus mucronatus L., Sp. Pl.: 50 (1753).

Schoenoplectus mucronatus (L.) Palla, Bot. Jahrb. Syst. 10: 299 (1888).

Schoenoplectiella supina (L.) Lye, Lidia 6: 27 (2003)

Scirpus supinus L., Sp. Pl.: 49 (1753).

Schoenoplectus supinus (L.) Palla, Bot. Jahrb. Syst. 10: 299 (1888).

Schoenoplectus (Rchb.) Palla, Verh. K. K. Zool.-Bot.

Ges. Wien 38 (Sitzungsber.): 49 (1888), nom. cons.

Schoenoplectus lacustris (L.) Palla, Bot. Jahrb. Syst. 10: 299 (1888).

Schoenoplectus lacustris subsp. lacustris

Scirpus lacustris L., Sp. Pl.: 48 (1753).

Schoenoplectus lacustris subsp. hippolyti (V. I. Krecz.) Kukkonen, Ann.

Naturhist. Mus. Wien, B 98B(Suppl.): 89 (1996).

Scirpus hippolyti V. I. Krecz., Bot. Mater. Gerb. Bot. Inst. Komarova Akad. Nauk S.S.S.R. 7: 28 (1937).

Schoenoplectus hippolyti (V. I. Krecz.) V. I. Krecz. ex Grossh., Fl. Kavkaza, ed. 2, 2: 16(1940).

Schoenoplectus grossheimii Pobed., Bot. Mater. Gerb. Bot. Inst. Komarova Akad. Nauk S.S.S.R. 12: 17 (1950).

Scirpus grossheimii (Pobed.) Czerep., Sosud. Rast. SSSR: 207 (1981).

Schoenoplectus litoralis (Schrad.) Palla, Bot. Jahrb. Syst. 10: 299 (1888) Scirpus litoralis Schrad., Fl. Germ. 1: 142 (1806).

Schoenoplectus pungens (Vahl) Palla, Bot. Jahrb. Syst. 10: 299 (1888). Scirpus pungens Vahl, Enum. Pl. Obs. 2: 255 (1805).

Schoenoplectus tabernaemontani (C. C. Gmel.) Palla, Bot. Jahrb. Syst. 10: 299 (1888).

Scirpus tabernaemontani C. C. Gmel., Fl. Bad. 1: 101 (1805).

Scirpus lacustris L. subsp. tabernaemontani (C. C. Gmel.) Syme in J. E. Smith, Engl. Bot., ed. 3a, 10: 64 (1870).

Schoenoplectus lacustris (L.) Palla subsp. tabernaemontani (C. C. Gmel.) Á. Löve \& D. Löve, Folia Geobot. Phytotax. 10: 275 (1975).

Schoenoplectus triqueter (L.) Palla, Bot. Jahrb. Syst. 10: 299 (1888). Scirpus triqueter L., Mant. Pl. 1: 29 (1767).

Schoenus L., Sp. Pl.: 42 (1753).

Schoenus ferrugineus L., Sp. Pl.: 43 (1753)

Schoenus nigricans L., Sp. Pl.: 43 (1753).
Scirpoides holoschoenus (L.) Soják, Čas. Nár. Mus., Odd. Prír. 140: 127 (1972).

Scirpus holoschoenus L., Sp. Pl.: 49 (1753).

Scirpus romanus L., Sp. Pl.: 49 (1753).

Scirpus australis L., Syst. Veg. ed. 13: 85 (1774).

Holoschoenus vulgaris Link, Hort. Berol. 1: 293 (1827).

Holoschoenus australis (L.) Rchb., Flora 13: 499 (1830).

Holoschoenus romanus (L.) Fritsch, Exkursionsfl. Österreich.: 80 (1897).

Scirpoides holoschoenus (L.) Soják subsp. australis (L.) Soják, Čas. Nár. Mus., Odd. Prír. 141: 61 (1972).

Scirpoides romana (L.) Soják, Čas. Nár. Mus., Odd. Prír. 140: 127 (1972).

Scirpus Tourn. ex L., Sp. Pl.: 51 (1753), nom. cons.

Scirpus radicans Schkuhr, Ann. Bot. (Usteri) 4: 49 (1793).

Scirpus sylvaticus L. var. radicans (Schkuhr) Willd., Sp. Pl., ed. 4, 1: 308 (1797).

Scirpus sylvaticus L., Sp. Pl.: 51 (1753).

Trichophorum Pers., Syn. Pl. 1: 69 (1805).

Trichophorum cespitosum (L.) Hartm. Handb. Skand. Fl., ed. 5: 259 (1849). Scirpus cespitosus L., Sp. Pl.: 48 (1753).

Trichophorum alpinum (L.) Pers., Syn. Pl. 1: 70 (1805).

Eriophorum alpinum L., Sp. Pl.: 53 (1753).

Eleocharis alpina (L.) Bluff, Nees \& Schauer, Comp. Fl. German. 1(1): 92 (1836).

Baeothryon alpinum (L.) T. V. Egorova, Novosti Sist. Vyssh. Rast. 8: 85 (1971).

Family Juncaceae Juss.

Juncus L., Sp. Pl.: 325 (1753).

Juncus acutiflorus Ehrh. ex Hoffm., Deutschland Flora: 125 (1791).

Juncus acutiflorus subsp. acutiflorus

Juncus alpinoarticulatus Chaix, Pl. Vapinc.: 74 (1785) \& Chaix, in Vill.,

Hist. Pl. Dauphiné (Villars) 1: 378 (1786).

Juncus alpinus Vill., Hist. Pl. Dauphiné (Villars) 2: 233 (1787), nom. illeg.

Juncus alpinoarticulatus subsp. alpinoarticulatus

Juncus alpinus f. carpaticus Racib. Spraw. Komis. Fizjogr. 22(2): $165(1888)$

Juncus carpaticus Simonk., Term. Füz. 10: 184 (1886).

Juncus carpaticus f. minor Zapał., Consp. Fl. Galic. 1: 136 (1906).

Juncus fuscoater Schreb., in Schweigg. \& Koerte, Fl. Erlang. 149

(1811).

Juncus geniculatus auct. non Schrank

Juncus articulatus L., Sp. Pl.: 327 (1753).

Juncus articulatus subsp. articulatus

Juncus adscendens Host, Icon. Descr. Gram. Austriac. 3: 58 (1805), nom. illeg.

Juncus geniculatus Schrank, Baier. Fl. 1: 613 (1789).

Juncus lampocarpus Ehrh. ex Hoffm., Deutschl. Fl., ed. 2, 1: 166 (1800).

Juncus macrocephalus Viv., Fl. Cors. Prodr.: 5 (1824).

Juncus articulatus var. nigrescens Lindem., Prodr. Fl. Cherson.: 206 (1872).

Juncus articulatus var. pallescens Lindem., Prodr. Fl. Cherson.: 206 (1872).

Juncus lampocarpus f. nigrescens Zapał., Consp. Fl. Galic. 1: 138 (1906).

Juncus articulatus f. nigrescens (Zapał.) Sóo, Acta Bot. Acad. Sci. Hung. 16: 366 (1971).

Juncus atratus Krock., Fl. Siles. (Krocker) 1: 562 (1787). 
Juncus acutiflorus Griseb., non Ehrh. ex Hoffm., Spic. Fl. Rumel. 2(5/6): 407 (1846).

Juncus atratus f. fuscescens Racib., Spraw. Komis. Fizjogr. 22(2): 167 (1888).

Juncus bufonius L., Sp. P1.: 328 (1753).

Juncus bufonius var. dolichophyllus Zapał., Consp. Fl. Galic. 1: 129 (1906).

Juncus erythropodus V. I. Krecz., Bull. Univ. As. Med. 21: 176 (1935).

Juncus juzepczukii V. I. Krecz. \& Gontsch., Fl. URSS 3: 625 (1935).

Juncus bufonius f. minutulus Albert \& Jahand., Cat. Pl. Vasc. Var: 501 (1908).

Juncus minutulus (Albert \& Jahand) Prain, Index Kew. Suppl. 5: 143 (1921).

Juncus minutulus V. I. Krecz. \& Gontsch., Fl. URSS 3: 625 (1935), nom. illeg.

Juncus bufonius subsp. minutulus (V. I. Krecz. \& Gontsch.) Soó, Acta Bot. Acad. Sci. Hung. 16: 366 (1971), nom. illeg.

Juncus bulbosus L., Sp. Pl.: 327 (1753).

Juncus supinus Moench, Enum. Pl. Hass. 1: 167. (1777).

Juncus uliginosus Roth, Bot. Mag. (Römer \& Usteri) 1(2): tes St. 16 (1787).

Juncus capitatus Weigel, Observ. Bot. (Weigel): 28 (1772).

Juncus ericetorum Pollich, Hist. Pl. Palat. 1: 351 (1776).

Juncus gracilis Roth, Bot. Abh. Beobacht:: 46 (1788).

Juncus castaneus Smith, Fl. Brit. 1: 383 (1800).

Juncus castaneus subsp. castaneus

Juncus czetzii Schur, Oesterr. Bot. Z. 13: 111 (1863).

Juncus compressus Jacq., Enum. Stirp. Vindob.: 60, 235 (1762).

Juncus conglomeratus L., Sp. P1.: 326 (1753).

Juncus communis subsp. conglomeratus (L.) Čelak., Prodr. Fl. Böhm. 1: 80 (1867), nom. inval.

Juncus leersii T. Marsson, Fl. Neu-Vorpommern: 451 (1869).

*Juncus dichotomus Elliott, Sketch Bot. S. Carolina [Elliott] 1: 406 (1817). Juncus tenuis var. dichotomus (Elliott) Alph.Wood, Class-book Bot.: $726(1861)$.

Juncus tenuis subsp. dichotomus (Elliott) Verloove \& Lambinon, New J. Bot. 1(1): 40 (2011).

Juncus dichotomus var. platyphyllus Wiegland, Bull. Torrey Bot. Club 30: 448 (1903).

Juncus platyphyllus (Wiegland) Fernald, Rhodora 47: 124 (1945).

Juncus effusus L., Sp. Pl.: 326 (1753).

Juncus effusus subsp. effusus

Juncus communis E. Mey., Junci Gen. Monogr. Specim. 20 (1819), nom. illeg.

Juncus communis subsp. effusus (L.) Čelak., Prodr. Fl. Böhm. 1: 80 (1867), nom. inval.

Juncus filiformis L., Sp. P1.: 326 (1753).

Juncus transsilvanicus Schur, Verh. Mitth. Siebenbürg. Vereins Naturwiss. Hermannstadt 4: 77 (1853).

Juncus gerardii Loisel., in Desv. Journ. Bot. 2: 284 (1809).

Juncus gerardii subsp. gerardii

Juncus inflexus L., Sp. P1.: 326 (1753).

Juncus inflexus subsp. inflexus Juncus glaucus Sibth., Fl. Oxon.: 113 (1794), nom. illeg. Juncus glaucus Ehrh., Beitr. Naturk. [Ehrhart] 6: 83 (1791), nom. inval. Juncus glaucus var. macrocarpus Zapał., Consp. Fl. Galic. 1: 133 (1906).

Juncus paniculatus Hoppe ex Mert. \& W. D. J. Koch, Deutschl. Fl. (Mertens \& W. D. J. Koch), ed. 3. 2: 575, in obs. (1826).

Juncus littoralis C. A. Mey., Verz. Pfl. Casp. Meer.: 34 (1831). Juncus acuto-maritimus E. Mey., in C. F. von Ledebour, Fl. Ross. 4: 234 (1853), nom. illeg.

Juncus tommasinii Parl., Fl. Ital. (Parlatore) 2(2): 315 (1857).

Juncus acutus var. tyraicus Pacz., Fl. Chers. 1: 381 (1914).

Juncus tyraicus (Pacz.) V. I. Krecz. \& Gontsch., in V. L. Komarov, Fl. URSS 3: 544 (1935).

Juncus acutus subsp. tyraicus (Pacz.) I. Grint., in Savulescu, Fl. Reipubl. Popul. Roman. 11: 556 (1966).
Juncus littoralis subsp. tyraicus (Pacz.) Novikov, Fl. Evropeiskoi Chasti SSSR 2: 73 (1976).

Juncus maritimus Lam., Encycl. 3: 264 (1789).

Juncus ponticus Steven, Bull. Soc. Imp. Naturalistes Moscou 3: 94 (1857).

Juncus maritimus var. ponticus (Steven) Asch. \& Graebn., Syn. Mitteleur. Fl. 2(2): 456 (1904).

Juncus ranarius Songeon \& E. P. Perrier, in Billot, Annot. Fl. Fr. et Allem.: 192 (1860).

Juncus bufonius var. ranarius (Songeon \& E. P. Perrier) Farw., Rep. (Annual) Michigan Acad. Sci. 19: 247 (1917).

Juncus bufonius subsp. ranarius (Songeon \& E. P. Perrier) Hiitonen, Suom. Putkilok. (Enum. Pl. Vasc. Fenn. Or.): 22 (1934).

Juncus ambiguus auct. non Guss., Fl. Sicul. Prodr. 1: 435 (1827).

Juncus nastanthus V. I. Krecz. \& Gontsch., Fl. URSS 3: 517, 624 (1935).

Juncus bufonius subsp. nastanthus (V. I. Krecz. \& Gontsch.) Soó, Acta Bot. Acad. Sci. Hung. 16(3-4): 366 (1971).

Juncus soranthus Schrenk, Bull. Cl. Phys.-Math. Acad. Imp. Sci. SaintPétersbourg 2: 193 (1843).

Juncus gerardii subsp. soranthus (Schrenk) K. Richt., Pl. Eur. 1: 177 (1890).

Juncus gerardii var. soranthus (Schrenk) Trautv., Bull. Soc. Imp. Naturalistes Moscou 40(2): 110 (1867).

Juncus fominii Zoz, in Sympos. Mem. Fomin (Acad. Sc. Ukraine): 51 (1938).

Juncus sphaerocarpus Nees ex Funk, Flora 1: 521 (1818).

Juncus squarrosus L., Sp. Pl.: 327 (1753).

Juncus subnodulosus Schrank, Baier. Fl. i.: 616 (1789).

Juncus erectus Besser, Prim. Fl. Galiciae Austriac. 1: 241 (1809).

Juncus obtusatus Kit. ex Schult., Österreichs Fl. 1: 566 (1814).

Juncus obtusiflorus Ehrh., Beitr. Naturk. [Ehrhart] 6: 83 (1791).

Juncus sylvaticus (L.) Reichard, Fl. Moeno-Francof. 2: 181 (1778).

Juncus tenageia Ehrh. ex L.f., Suppl. P1.: 208 (1782).

Juncus tenageia subsp. tenageia

*Juncus tenuis Willd., Sp. Pl., ed. 4 [Willdenow] 2(1): 214 (1799).

Juncus macer Gray, Nat. Arr. Brit. Pl. 2: 164 (1821).

Juncus thomasii Ten., Ind. Sem. Hort. Neap.: 3 (1827).

Juncus thomasii var. laxiusculus Zapał. Consp. Fl. Gallic. Crit. 1: 135 (1906).

Juncus rochelianus Schult. \& Schult.f., Syst. Veg., ed. 15 bis [Roemer \& Schultes] 7(2): 1658 (1830).

Juncus trighumis L., Sp. Pl.: 328 (1753).

Juncus triglumis subsp. triglumis

Luzula DC., Fl. Franc. [de Candolle \& Lamarck], ed. 3. 3: 158 (1805), nom. cons.

Luzula alpinopilosa (Chaix) Breistr., Bull. Soc. Sci. Isère 61: 609 (1947). Juncus alpinopilosus Chaix, in D. Villars, Hist. Pl. Dauphiné 1: 318 (1786).

Luzula spadicea DC., in Lam. \& DC., Fl. Franç., ed. 3, 3: 159 (1805).

Luzula alpinopilosa subsp. obscura S. E. Fröhner, Preslia 40: 426 (1968).

Luzula obscura (S. E. Fröhner) Novikov, Byull. Moskovsk. Obshch. Isp. Prir., Otd. Biol. 95(6): 66 (1990).

Luzula carpatica Kitt. ex Kanitz, Linnaea 32: 327 (1863).

Luzula spadicea var. carpatica (Kitt. ex Kanitz) Asch. \& Graebn., Syn. Mitteleur. Fl. 2(2): 513 (1904).

Luzula spadicea f. carpatica (Kitt. ex Kanitz) I. Grint., Fl. Reipubl. Popul. Roman. 11: 594 (1966).

Luzula campestris (L.) DC., Fl. Franc. [de Candolle \& Lamarck], ed. 3, 3: 161 (1805).

Luzula campestris subsp. campestris

Juncus campestris L., Sp. Pl. 1: 329 (1753).

Luzula subpilosa (Gilib.) V. I. Krecz. \& Gontsch., Fl. URSS 3: 571 (1935), nom. illeg.

Luzula divulgata Kirschner, Folia Geobot. Phytotax. 14: 431 (1979 publ. 1980).

Luzula forsteri (Smith) DC., Syn. Pl. Fl. Gall.: 150 (1806). 
Juncus forsteri Sm., Fl. Brit. 3: 1395 (1804).

Luzula vernalis subsp. forsteri (Sm.) Bonnier \& Layens, Tabl. Syn. Pl. Vasc. France: 322 (1894).

Luzula forsteri subsp. forsteri

Luzula forsteri subsp. rhizomata (Ebinger) Z. Kaplan, Preslia 73: 60 (2001).

Luzula forsteri var. rhizomata Ebinger, Mem. New York Bot. Gard. 10(5): 289 (1964).

Luzula luzulina (Vill.) Racib., Spraw. Komis. Fizjogr. 22: 169 (1888).

Juncus flavescens Host, Icon. Descr. Gram. Austriac. 3: 62 (1805).

Juncus luzulinus Vill., Hist. Pl. Dauphiné 2: 235 (1787).

Luzula flavescens (Host) Gaudin, Agrost. Helv. 2: 239 (1811).

Luzula hostii Desv., J. Bot. (Desvaux) 1: 140 (1808), nom. superfl.

Luzula luzuloides (Lam.) Dandy \& Wilmott, Dandy \& E.Willm., J. Bot. 76: 352 (1938).

Juncus luzuloides Lam., Encycl. 3: 272 (1789).

Luzula luzuloides subsp. luzuloides

Juncus albidus Hoffm., Deutschl. Fl. (Hoffm.): 126, t. 6 (1791).

Luzula albida (Hoffm.) DC., Fl. Franc. [de Candolle \& Lamarck], ed. 3. 3: 159 (1805).

Luzula albida var. parviflora Döll., Fl. Baden, 1: 323 (1857).

Luzula angustifolia Wender, Beitr. Fl. Hess.: 36 (1823), nom. illeg.

Juncus nemorosus Pollich, Hist. Pl. Palat. 1: 352 (1776).

Luzula nemorosa (Pollich) E.Mey., Linnaea 22(4): 394, nom. illeg.

Luzula nemorosa var. leucanthema (Wallr.) Asch. \& Graebn., Syn. Mitteleur. Fl. 2(2): 502 (1904).

Luzula luzuloides subsp. rubella (Hoppe ex Mert. \& W. D. J. Koch)

Holub, Folia Geobot. Phytotax. 18: 205 (1983).

Luzula albida var. rubella Hoppe ex Mert. \& W. D. J. Koch in J. C. Röhling, Deutschl. Fl., ed. 3, 2: 599 (1826).

Luzula angustifolia subsp. rubella (Hoppe ex Mert. \& W. D. J. Koch) K. Richt., Pl. Eur. 1: 184 (1890).

Luzula nemorosa var. rubella (Hoppe ex Mert. \& W. D. J. Koch) Garcke, Fl. N. Mitt.-Deutschland, ed. 2: 340 (1851).

Luzula angustifolia var. rubella (Hoppe ex Mert. \& W. D. J. Koch) Garcke, Fl. N. Mitt.-Deutschland, ed. 3: 348 (1853).

Luzula nemorosa f. rubella (Hoppe ex Mert. \& W. D. J. Koch) Racib., Consp. Juncac. Polon.: 25 (1888).

Luzula fuscata Schur, Verh. Mitth. Siebenbürg. Vereins Naturwiss. Hermannstadt 10: 200 (1859).

Luzula alpigena Schur, Enum. Pl. Transsilv.: 681 (1866).

Luzula fuscata Schur, Verh. Mitth. Siebenbürg. Vereins Naturwiss. Hermannstadt 10: 200 (1859).

Luzula albida var. alpigena (Schur) Nyman, Consp. Fl. Eur.: 751 (1882).

Luzula albida var. cuprina Rochel. Pl. Banat. Rar. 3: 27 (1828), nom. inval.

Luzula nemorosa var. cuprina Rochel. ex Asch. \& Graebn., Syn. Mitteleur. Fl. 2(2): 503 (1904).

Luzula luzuloides subsp. cuprina (Roch. ex Asch. \& Graeb.) Chrtek \& Kř́sa, Preslia 46(3): 212 (1974).

Luzula multiflora (Ehrh.) Lej., Fl. Spa 1: 169 (1811).

Luzula multiflora subsp. multiflora

Juncus campestris var. multiflorus Ehrh., Beitr. Naturk. 5: 14 (1790).

Juncus multiflorus (Ehrh.) Hoffm., Deutschl. Fl., ed. 3, 1: 169 (1800), nom. illeg.

Juncus nemorosus Host, Icon. Descr. Gram. Austriac. 3: 64 (1805), nom. illeg.

Luzula nemorosa Hornem., Hort. Bot. Hafn.: 962 (1815).

Luzula campestris var. nemorosa (Hornem.) Gaudin, Fl. Helv. 2: 572 (1828).

Luzula pallescens Sw., Summa Veg. Scand.: 13 (1814).

Juncus campestris var. lapponicus Retz., Fl. Scand. Prodr., ed. 2: 82 (1795).

Juncus pallescens Wahlenb., Fl. Lapp.: 87 (1812), nom. illeg.

Luzula campestris subsp. pallescens (Sw.) Čelak., Prodr. Fl. Böhmen 4: 749 (1881).

Luzula multiflora subsp. pallescens (Sw.) Reichg., in S. J. van Ooststroom et al., Fl. Neerl. 1: 208 (1964).
Luzula pallidula Kirschner, Taxon 39: 110 (1990).

Luzula pilosa (L.) Willd., Enum. Pl. 393 (1809).

Juncus pilosus L., Sp. Pl.: 329 (1753).

Juncus pilosus var. vernalis (Reichard) Pers., Syn. Pl. 1: 385 (1805).

Juncus vernalis Reichard, Fl. Moeno-Francof. 2: 182 (1778).

Luzula vernalis (Reichard) DC., Fl. Franc. [de Candolle \& Lamarck], ed. 3, 3: 160 (1805).

Luzula spicata (L.) DC., Fl. Franc. [de Candolle \& Lamarck], ed. 3, 3: 161 (1805).

Juncus spicatus L., Sp. Pl.: 330 (1753).

Luzula spicata subsp. conglomerata (W. D. J. Koch) Murr, Magyar Bot. Lapok 28: 67 (1929 publ. 1930).

Luzula glomerata Miel. ex Huebener, Flora 22(2): 489 (1839).

Luzula spicata var. conglomerata W. D. J. Koch, Syn. Fl. Germ. Helv., ed. 2: 848 (1844).

Luzula spicata subsp. mutabilis Chrtek \& Kř́sa, Bot. Not. 115: 302 (1962).

Luzula sudetica (Willd.) Schult., Oesterr. Fl., ed. 2, 1: 573 (1814).

Juncus sudeticus Willd., Sp. Pl. 2: 221 (1799).

Luzula campestris var. nigricans Gaudin, Fl. Helv. 2: 572 (1828).

Luzula campestris subsp. alpestris (G.Mey.) Čelak., Prodr. Fl. Böhmen 1: 85 (1867).

Luzula campestris subsp. sudetica (Willd.) Čelak., Prodr. Fl. Böhmen 3: 749 (1881), nom. illeg.

Luzula multiflora subsp. sudetica (Willd.) Arcang., Comp. Fl. Ital.: 713 (1882), nom. illeg.

Luzula althii Herbich, Sel. Pl. Galic.: 12 (1836).

Luzula sylvatica (Huds.) Gaudin, Agrost. Helv. 2: 240 (1811).

Luzula sylvatica subsp. sylvatica

Juncus sylvaticus Huds., Fl. Angl.: 32 (1762).

Luzula maxima (Reichard) DC., Fl. Franc. [de Candolle \& Lamarck], ed. 3. 3: 160 (1805).

Luzula taurica (V. I. Krecz.) Novikov, Novosti Sist. Vyssh. Rast. 27: 20 (1990).

Luzula campestris subsp. taurica V. I. Krecz. Zhurn. Russk. Bot. Obshch. 12: 490 (1928).

Luzula multiflora subsp. taurica (V. I. Krecz.) Novikov, Fl. Evropeiskoi Chasti SSSR 2: 83 (1976).

Oreojuncus Záv. Drábk. \& Kirschner, Preslia 85: 498 (2013)

Oreojuncus trifidus (L.) Záv. Drábk. \& Kirschner, Preslia 85(4): 499 (2013).

Juncus trifidus L., Sp. Pl.: 326 (1753).

\section{Discussion}

To the list of Juncales in the flora of Ukraine, we included its recent recorded taxa and some taxa unconfirmed by reliable herbarium data (for the family Cyperaceae: Carex (Hynda \& Danylyk, 1994; Danylyk, 1995; Danylyk \& Panchenko, 2001), Cyperus (= Torulinium) (Dubyna \& Protopopova, 1984), Eleocharis (Danylyk \& Orlov, 2004), Eriophorum (Danylyk, 2004), Trichophorum (Danylyk et al., 2007; Kuzyarin, 2012), Schoenoplectus (Danylyk \& Honcharenko, 2009), Schoenoplectiella (Danylyk et al., 2017, Moysiyenko et al., 2019) and for the family Juncaceae: Juncus (Olshanskyi \& Orlov, 2013; Shevchyk et al., 2018)).

The results of the recent phylogenetic studies (Global Carex Group, 2015, 2016; Shiels et al., 2014; Záveská Drábková \& Kirschner, 2013) have somewhat changed our understanding of the taxonomy of Juncales and some taxa of this order. Compared to previous researches, we accepted genera Schoenoplectiella Lye and Oreojuncus Záv. Drábk. \& Kirschner. In our work, the basic unit is the species. When necessary we also indicated subspecies, and varieties, and forms to cover intraspecific heterogeneity.

The order Juncales in Ukraine contains 188 species, belonging to two families (Cyperaceae, and Juncaceae), and 19 genera, (Table 1). Within the genera of the order Juncales very different number of species has been identified - from 1 to 96 species. Some genera (Blysmus, Cladium, Fimbristylis, Isolepis, Rhynchospora, Scirpoides, and Oreojuncus) include 
only one species. Five genera contain more than ten species each, namely Carex contains 96 species, Juncus - 26 species, Luzula - 13 species, Cyperus - 12 species, Eleocharis - 11 species. Other genera of the order Juncales are represented in the flora of Ukraine by two to five species.

Table 1

The order Juncales in Ukraine: taxonomical diversity

\begin{tabular}{|c|c|}
\hline Taxon & Namber of species \\
\hline Cyperaceae & 148 \\
\hline Blysmus & 1 \\
\hline Bolboschoenus & 5 \\
\hline Carex & 96 \\
\hline Cladium & 1 \\
\hline Cyperus & 12 \\
\hline Eleocharis & 11 \\
\hline Eriophorum & 4 \\
\hline Fimbristylis & 1 \\
\hline Isolepis & 1 \\
\hline Rhynchospora & 1 \\
\hline Schoenoplectiella & 3 \\
\hline Schoenoplectus & 5 \\
\hline Schoenus & 2 \\
\hline Scirpoides & 1 \\
\hline Scirpus & 2 \\
\hline Trichophorum & 2 \\
\hline Juncaceae & 40 \\
\hline Juncus & 26 \\
\hline Luzula & 13 \\
\hline Oreojuncus & 1 \\
\hline
\end{tabular}

The number of Juncales species is greater in Ukraine than in adjacent European countries (please see Table 2). In the neighbouring countries, the number of Juncales species is as follows:

- Cyperaceae: Poland - 146 (Mirek et al., 2002), Belarus - 103 (Skuratovich, 2017), Moldova - 75 (Geydeman, 1986), Hungary - 112 (Lájer, 2009), Slovakia - 137 (Dostal, 1989), Romania - (Ciocârlan, 2009);

- Juncaceae: Poland - 34 (Kirschner, 1979, 1992; Mirek et al., 2002), Belarus - 28 (Dubovik \& Tretjakov, 2017), Moldova - 17 (Geydeman, 1986), Hungary - 24 (Lájer, 2009), Slovakia - 33 (Dostal, 1989), Romania - 32 (Grinţescu, 1966; Ciocârlan, 2009).

Quantitative taxonomic indicators of Juncales of the flora of Ukraine are closer to the countries with mountainous regions (Poland, Romania, Slovakia). In Ukraine, the number of Juncales taxa is significantly higher compared to countries where there are no mountains (Moldova, Belarus, Hungary). In general, the degree of taxonomic diversity of Juncales in Ukraine is quite high compared to the neighbouring countries (Fig. 1).

\section{Table 2}

Number of Juncales species in Ukraine and adjacent countries

\begin{tabular}{cccccccc}
\hline \multirow{2}{*}{ Taxon } & \multicolumn{7}{c}{ Country } \\
\cline { 2 - 8 } & Ukraine & Belarus & Poland & Slovakia & Hungary & Romania & Moldova \\
\hline Cyperaceae & 148 & 103 & 141 & 137 & 112 & 146 & 75 \\
Juncaceae & 40 & 28 & 34 & 33 & 24 & 32 & 17 \\
Total & 188 & 131 & 175 & 170 & 136 & 178 & 92 \\
\hline
\end{tabular}

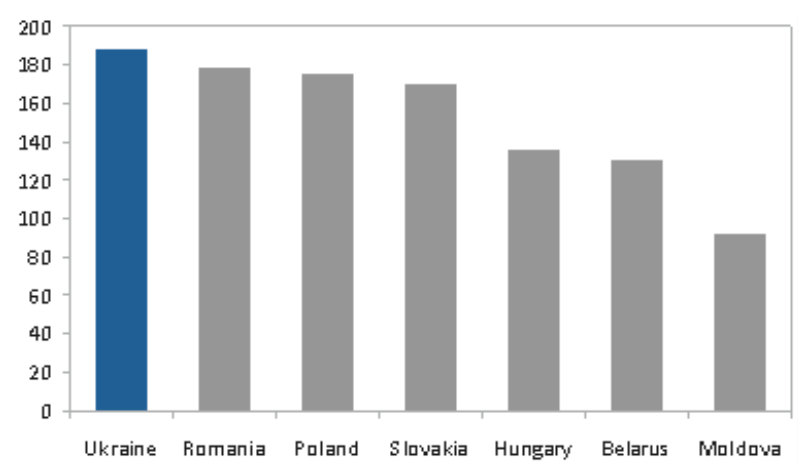

Fig. 1. Number of Juncales species in Ukraine and adjacent countries

Thus, the order Juncales has a great taxonomic diversity in Ukraine. Compared with the neighbouring countries, the number of species of this order is quite high. This is ensured by the special geographical position of
Ukraine (primarily due to the considerable length from north to south, as well as the presence of mountains).

In the territory of Ukraine, there are both widespread and rare Juncales species. We refer to the rarest species Carex alba, C. bicolor, C. bohemica, C. brunnescens, C. buxbaumii, C. depauperata, C. fuliginosa, C. globularis, C. heleonastes, C. lachenalii, C. loliacea, C. obtusata (Sosnovska et al., 2013), C. pediformis, C. rupestris, C. strigosa, Cyperus longus, Eleocharis multicaulis, E. oxylepis, Fimbristylis bisumbellata, Juncus acutiflorus, J. soranthus, J. subnodulosus, Isolepis setacea, Luzula spicata, Schoenoplectus pungens, Trichophorum alpinum, T. cespitosum. Also, the following species are rare: Bolboschoenus yagara, Carex chordorrhiza, C. davalliana, C. dioica (Sosnovska et al., 2017), C. hostiana, C. pauciflora, C. secalina, C. vaginata, Cladium mariscus, Eleocharis carniolica, Juncus capitatus, J. castaneus, J. littoralis, J. sphaerocarpus, J. thomasii, J. triglumis, Luzula alpinopilosa, L. sudetica, Schoenoplectiella mucronata, Schoenus ferrugineus. Perhaps, two species (Juncus tenageia, Luzula luzulina) have disappeared in Ukraine.

Three Juncales species are non-native to Ukraine: Cyperus esculentus, Juncus dichotomus, J. tenuis.

\section{Conclusions}

Thus, we have compiled an updated list of Juncales species in the flora of Ukraine. There are 188 species of order Juncales in Ukraine, belonging to families Cyperaceae and Juncaceae, and 19 genera. The genus Carex is the largest in this order. Seven genera have only one species each. The genus Juncus includes 26 species, Luzula - 13 species, Cyperus 12 species, Eleocharis - 11 species. Other genera of the Juncales are represented in the flora of Ukraine by two to five species. This is a fairly high rate of taxonomic diversity compared with the countries adjacent to Ukraine.

\section{References}

APG IV (2016). An update of the Angiosperm Phylogeny Group classification for the orders and families of flowering plants: APG IV. Botanical Joumal of the Linnean Society, 181(1), 1-20.

Bačič, T., Jogan, N., \& Dolenc Koce, J. (2007). Luzula sect. Luzula in the SouthEastern Alps: Karyology and genome size. Taxon, 56, 129-136.

Bartolucci, F., Peruzzi, L., Galasso, G., Albano, A., Alessandrini, A., Ardenghi, N. M. G., Astuti, G., Bacchetta, G., Ballelli, S., Banfi, E., Barberis, G., Bernardo, L., Bouvet, D., Bovio, M., Cecchi, L., Di Pietro, R., Domina, G., Fascetti, S., Fenu, G., Festi, F., Foggi, B., Gallo, L., Gottschlich, G., Gubellini, L., Iamonico, D., Iberite, M., Jiménez-Mejías, P., Lattanzi, E., Marchetti, D., Martinetto, E., Masin, R. R., Medagli, P., Passalacqua, N. G., Peccenini, S., Pennesi, R., Pierini, B., Poldini, L., Prosser, F., Raimondo, F. M., Roma-Marzio, F., Rosati, L., Santangelo, A., Scoppola, A., Scortegagna, S., Selvaggi, A., Selvi, F., Soldano, A., Stinca, A., Wagensommer, R. P., Wilhalm, T., \& Conti, F. (2018). An updated checklist of the vascular flora native to Italy. Plant Biosystems, 152(2), 179-303.

Bozek, M., Leitch, A. R., Leitch, I. J., Záveská Drábková, L., \& Kuta, E. (2012) Chromosome and genome size variation in Luzula (Juncaceae), a genus with holocentric chromosomes. Botanical Journal of the Linnean Society, 170(4), $529-541$.

Bremer, K. (2002). Gondwanan evolution of the grass alliance of families (Poales). Evolution, 56(7), 1374-1387.

Ciocârlan, V. (2009). Flora Ilustrată a României: Pteridophyta et Spermatophyta. [The illustrated flora of Romania: Pteridophyta et Spermatophyta]. Ceres, Bucureşti.

Danylyk, I. M. (1995). Carex strigosa Huds. (Cyperaceae) - novyi vyd dlia flory Ukrainy [Carex strigosa Huds. (Cyperaceae) - the new species for the flora of Ukraine]. Ukrainian Botanical Journal, 52(2), 277-279 (in Ukrainian).

Danylyk, I. M. (2004). Rid Eriophorum L. (Cyperaceae) u flori Ukrainy [The genus Eriophorum L. (Cyperaceae) in the flora of Ukraine]. In: J. K. Pachoski and modern botany. Ailant, Kherson. Pp. 96-98 (in Ukrainian).

Danylyk, I. M. (2012). Systema rodyny Cyperaceae Juss. flory Ukrainy [A system of the family Cyperaceae Juss. in the Ukrainian flora]. Ukrainian Botanical Journal, 69(3), 337-351 (in Ukrainian).

Danylyk, I. M., \& Orlov, A. A. (2004). Novi znakhidky Eleocharis mamillata (Cyperaceae) na Pravoberezhnomu Polissi Ukrainy [New localities Eleocharis mamillata (Cyperaceae) in Right-Bank Polissya of Ukraine]. Ukrainian Botanical Journal, 61(5), 84-90 (in Ukrainian).

Danylyk, I. M., \& Panchenko, S. M. (2001). Carex brunnescens (Pers.) Poiret (Cyperaceae) - novyi vyd flory Ukrainy [Carex brunnescens (Pers.) Poiret (Cyperaceae) - a new species for the flora of Ukraine]. Ukrainian Botanical Journal, 58(1), 73-77 (in Ukrainan). 
Danylyk, I. M., \& Honcharenko, V. I. (2009). Schoenoplectus pungens (Vahl) Palla (Cyperaceae) - novyj vyd dlia flory Ukrainy [Schoenoplectus pungens (Vahl) Palla (Cyperaceae) - a new species for of the Ukrainian flora]. Ukrainian Botanical Journal, 66(5), 650-655 (in Ukrainan).

Danylyk, I. M., Myhaly, A. V., \& Kish, R. Y. (2007). Trichophorum Pers. (Cyperaceae) - novyj rid dlia flory Ukrainy [Trichophorum Pers. (Cyperaceae) - new genus for the Ukrainian flora]. Ukrainian Botanical Journal, 64(6), 905-909 (in Ukrainian).

Danylyk, I. M., Olshanskyi, I. G., \& Zhygalova, S. L. (2017). A new nomenclatural combination in Schoenoplectiella (Cyperaceae). Phytotaxa, 299(1), 138-140.

Ding, H.-B., Maw, M. B., Yang, B., Bouamanivong, S., \& Tan, Y.-H. (2020). An updated checklist of Begonia (Begoniaceae) in Laos, with two new species and five new records. In: Jin, X.-H., Xia, N.-H., \& Tan, Y.-H. (Eds.). Plant diversity of Southeast Asia-II. PhytoKeys, 138, 187-201.

Dostál, J. (1989). Nová Květena ČSSR [New Flora of Czechoslovakia]. Vol. 2. Akademia, Praha (in Czech)

Drábková, L., Kirschner, J., Seberg, O., Petersen, G., \& Vlček, Č. (2003). Phylogeny of the Juncaceae based on rbcL sequences, with special emphasis on Luzula DC. and Juncus L. Plant Systematics and Evolution, 240, 133-147.

Drábková, L., Kirschner, J., Vlček, Č., \& Pačes, V. (2004). trnL-trnF intergenic spacer and tmL intron define clades within Luzula and Juncus (Juncaceae). Journal of Molecular Evolution, 59, 1-10.

Drábková, L., Kirschner, J., \& Vlček, Č. (2006). Phylogenetic relationships within Luzula DC. and Juncus L. (Juncaceae): A comparison of phylogenetic signals of tmL-tmF intergenic spacer, trnL intron and rbcL plastome sequence data. Cladistics, 22, 132-143.

Dubovik, D. V., \& Tretyakov, D. I. (2017). Juncareae Juss. - sitnikovye. In: Parfenov, V. I. (Ed.). Flora Belarusi. Sosudistye rastenia [Flora of Belarus. Vascular Plants]. Vol. 3. Belaruskaya Navuka, Minsk. Pp. 310-351 (in Russian).

Dubyna, D. B., \& Protopopova, V. V. (1984). Novyi dlia flory evropeiskoyi chastyny SRSR vyd Torulinium ferax (Rich.) Urb. [Torulinium ferax (Rich.) Urb. a new species for the flora of the European part of the USSR]. Ukrainian Botanical Journal, 41(5), 21-25 (in Ukrainian).

Egorova, T. V. (1999). The sedges (Carex L.) of Russia and adjacent states (within the limits of the former USSR). Saint-Petersburg State Chemical-Pharmaceutical Academy, Saint-Petersburg; Missuri Botanical Garden Press, Saint Louis.

Egorova, T. V. (2004). Rod Scirpus L. (Cyperaceae) vo flore Eurasii [The genus Scirpus L. (Cyperaceae) in the flora of Eurasia]. Novosti Sistematiki Vysshikh Rastenii, 36, 40-79 (in Russian).

Egorova, T. V. (2005). Taxonomicheskiy obzor roda Schoenoplectus (Reihenb.) Palla (Cyperaceae) flory Severnoj Eurasii [A taxonomic review of the genus Schoenoplectus (Reihenb.) Palla (Cyperaceae) of the flora of Northem Eurasia]. Novosti Sistematiki Vysshikh Rastenii, 37, 49-79 (in Russian).

Egorova, T. V. (2007). Taxonomicheskiy obzor roda Eleocharis R. Br. (Cyperaceae) flory Evropy [Taxonomic review of the genus Eleocharis R. Br. (Cyperaceae) in the flora of Europe]. Novosti Sistematiki Vysshikh Rastenii, 39, 159-192 (in Russian)

Geydeman, T. S. (1986). Opredelitel vysshikh rasteniy Moldavskoy SSR [Key to higher plants of the Moldavian SSR]. Third edition. Știința, Chișinău (in Russian).

Global Carex Group (2015). Making Carex monophyletic (Cyperaceae, tribe Cariceae): A new broader circumscription. Botanical Journal of the Linnean Society, 179(1), 1-42.

Global Carex Group (2016). Megaphylogenetic specimen-level approaches to the Carex (Cyperaceae) phylogeny using ITS, ETS, and matK sequences: Implications for cassification. Systematic Botany, 41(3), 500-518.

Gonzalez-Elizondo, M. S., \& Peterson, P. M. (1997). A classification of and key to the supraspecific taxa in Eleocharis (Cyperaceae). Taxon, 46(3), 433-449.

Grinţescu, I. (1966). Juncaceae A. L. Juss. In: Flora Reipublicae Socialisticea România. Vol. 11. Pp. 527-604 (in Romanian).

Hayasaka, E. (2012). Delineation of Schoenoplectiella Lye (Cyperaceae), a genus newly segregated from Schoenoplectus (Rchb.) Palla. Japanese Journal of Botany, 87, 169-186.

Hroudova, Z., Zakravsky, P., Duchacek, M., \& Marhold, K. (2007). Taxonomy, distribution and ecology of Bolboschoenus in Europe. Annales Botanici Fennici, 44, 81-102.

Hynda, L. V., \& Danylyk, I. M. (1994). Carex demissa Homem. (Cyperaceae) - novyj vyd flory Ukrainy [Carex demissa Hornem. (Cyperaceae) - a new species of the flora of Ukraine]. Ukrainian Botanical Journal, 51(6), 134-137 (in Ukrainian).

Jones, E., Simpson, D. A., Hodkinson, T. R., Chase, M. W., \& Parnell, J. A. N. (2007). The Juncaceae-Cyperaceae interface: A combined plastid sequence analysis. Aliso, 23, 55-61.

Kirschner, J. (1979). A new species of the Luzula campestris-multiflora complex in Central Europe. Folia Geobotanica et Phytotaxonomica, 14, 431-435.

Kirschner, J. (1992). Karyological differentiation of Luzula sect. Luzula (Juncaceae) in Europe. Thaiszia - Journal of Botany, 2, 11-39.

Kirschner, J., Snogerup, S., Novikov, V. S., \& Ahti, T. (Eds.). (2002a). Species Plantarum: Flora of the World. Part 6. Juncaceae 1: Rostkovia to Luzula. Australian Biological Resources Study, Canberra.
Kirschner, J., Snogerup, S., Novikov, V. S., \& Ahti, T. (Eds.). (2002b). Species Plantarum: Flora of the World. Part 7. Juncaceae 2: Juncus subg. Juncus. Australian Biological Resources Study, Canberra.

Kirschner, J., Snogerup, S., Novikov, V. S., \& Ahti, T. (Eds.). (2002c). Species Plantarum: Flora of the World. Part 8. Juncaceae 3: Juncus subg. Agathryon. Australian Biological Resources Study, Canberra.

Koopman, J. (2015). Carex Europaea. The genus Carex L. (Cyperaceae) in Europe 1, ed. 2, ebook. Margraf Publishers, Weikersheim.

Kuzyarin, O. T. (2012). Trichophorum alpinum (L.) Pers. (Cyperaceae) - novyj vyd dlia flory Ukrayiny [Trichophorum alpinum (L.) Pers. (Cyperaceae), a new species in the flora of Ukraine]. Ukrainian Botanical Journal, 69(5), 708-712 (in Ukrainian).

Lájer, K. (2009). Juncaceae \& Cyperaceae. In: Király, G. Új Magyar füvészkönyv. Magyarország hajtásos növényei. Határozókulcsok [New Hungarian Herbal. The Vascular Plants of Hungary. Identification key]. Aggteleki Nemzeti Park Igazgatóság, Jósvafö. Pp. 493-570 (in Hungarian).

Larridon, I., Bauters, K., Reynders, M., Huygh, W., \& Goetghebeur, P. (2014). Taxonomic changes in $\mathrm{C}_{4}$ Cyperus (Cypereae, Cyperoideae, Cyperaceae): Combining the sedge genera Ascolepis, Kyllinga and Pycreus into Cyperus s.l. Phytotaxa, $166,33-48$.

Larridon, I., Bauters, K., Reynders, M., Huygh, W., Muasya, A. M., Simpson, D. A., \& Goetghebeur, P. (2013). Towards a new classification of the giant paraphyletic genus Cyperus (Cyperaceae): Phylogenetic relationships and generic delimitation in $\mathrm{C}_{4}$ Cyperus. Botanical Journal of the Linnean Society, 172, 106-126.

Larridon, I., Reynders, M., Huygh, W., Bauters, K., Vrijdaghs, A., Leroux, O., Muasya, A. M., Simpson, D. A., \& Goetghebeur, P. (2011). Taxonomic changes in $\mathrm{C}_{3}$ Cyperus (Cyperaceae) supported by molecular data, morphology, embryography, ontogeny and anatomy. Plant Ecology and Evolution, 144, 327-356.

Mirek, Z., Piękoś-Mirkowa, H, Zając, A., \& Zając, M. (2002). Flowering plants and pteridophytes of Poland. A checklist. Biodiversity of Poland. Vol. 1. W. Szafer Institute of Botany, Kraków.

Moysiyenko, I. I., Danylyk, I. M., Melnyk, R. P., Sadova, O. F., \& Zakharova, M. Y. (2019). Schoenoplectiella mucronata (Cyperaceae) u flori Ukrajiny [Schoenoplectiella mucronata (Cyperaceae) in the flora of Ukraine]. Ukrainian Botanical Journal, 76(1), 52-59 (in Ukrainian).

Mosyakin, S. L. (2013). Rodyny i poriadky kvitkovykh roslyn flory Ukrajiny: Pragmatychna klasyfikacia ta polozhennia u filogenetychnij systemi [Families and orders of angiosperms of the flora of Ukraine: A pragmatic classification and placement in the phylogenetic system]. Ukrainian Botanical Journal, 70(3), 289-307 (in Ukrainian).

Mosyakin, S. L., \& Fedoronchuk, M. M. (1999). Vascular plants of Ukraine. A nomenclatural checklist. M. G. Kholodny Institute of Botany, Kyiv.

Muasya, A. M., Simpson, D. A., \& Chase, M. W. (2001). A phylogeny of Isolepis (Cyperaceae) inferred using plastid rbcL and trnL-F sequences data. Systematic Botany, 26, 342-353.

Muasya, A. M., Simpson, D. A., Chase, M. W., \& Culham, A. (2000). Phylogenetic relationships within the heterogeneous Scirpus s.l. (Cyperaceae) inferred from rbcL and trnL-F sequence data. In: Wilson, K. L., \& Morrison, D. A. (Eds.). Monocots: Systematics and evolution. CSIRO, Melbourne. Pp. 610-614.

Munro, S. L., Kirschner, J., \& Linder, H. P. (2001). Species Plantarum: Flora of the World. Part 5. Prioniaceae. Australian Biological Resources Study, Canberra.

Novoselova, M. S. (2001). Rod Eriophorum L. (Cyperaceae) vo flore Rossii [The genus Eriophorum L. (Cyperaceae) in the flora of Russia]. Novosti Sistematiki Vysshikh Rastenii, 33, 44-55 (in Russian).

Novoselova, M. S. (2003). Rod Trichophorum Pers. (Cyperaceae) vo flore Rossii [The genus Trichophorum Pers. (Cyperaceae) in the flora of Russia]. Novosti Sistematiki Vysshikh Rastenii, 35, 41-50 (in Russian).

Olshanskyi, I. G., \& Fedoronchuk, M. M. (2011). Rid Juncus L. (Juncaceae) u flori Ukrayiny [Juncus L. (Juncaceae) in the Flora of Ukraine]. Ukrainian Botanical Journal, 68(5), 686-700 (in Ukrainian).

Olshanskyi, I. G., \& Fedoronchuk, M. M. (2012). Rid Luzula DC. (Juncaceae) u flori Ukrayiny [Luzula DC. (Juncaceae) in the Flora of Ukraine]. Ukrainian Botanical Journal, 69(1), 69-76 (in Ukrainian).

Olshanskyi, I. G., \& Orlov, O. O. (2013). Juncus dichotomus Elliott (Juncaceae), a new alien species for the flora of Ukraine. Ukrainian Botanical Journal, 70(6), 769-771.

Plunkett, G. M., Soltis, D. E., Soltis, P. S., \& Brooks, R. E. (1995). Phylogenetic relationships between Juncaceae and Cyperaceae: insights from rbcL sequence data. American Journal of Botany, 82(4), 520-525.

Reveal, J. L. (2012). An outline of a classification scheme for extant flowering plants. Phytoneuron, 37, 1-221.

Reyes-Chávez, J., Tarvin, S., \& Batke, S. P. (2021). Ferns and lycophytes of Honduras: A new annotated checklist. Phytotaxa, 506(1), 1-113.

Rojas-Alvarado, G., Blanco, M. A., \& Karremans, A. P. (2021). A taxonomic synopsis and morphological characterization of Myoxanthus (Orchidaceae: Pleurothallidinae). Phytotaxa, 507(3), 211-258.

Shevchyk, V. L., Olshanskyi, I. G., \& Senchylo, O. O. (2018). Juncus dichotomus Elliott (Juncaceae) - novyj adventyvnyj vyd roslyn dlia Zakarpattia [Juncus di- 
chotomus Elliott (Juncaceae) - new alien species for Transcarpathia]. Biolohichni Studii, 12(1), 135-139 (in Ukrainian)

Shiels, D. R., Hurlbut, D. L., Lichtenwald, S. K., \& Monfils, A. K. (2014). Monophyly and phylogeny of Schoenoplectus and Schoenoplectiella (Cyperaceae): Evidence from chloroplast and nuclear DNA sequences. Systematic Botany, 39(1), 132-144.

Skuratovich, A. N. (2017). Cyperaceae Juss. - osokovye. In: Parfenov, V. I. (Ed.) Flora Belarusi. Sosudistye rastenia [Flora of Belarus. Vascular Plants]. Vol. 3. Belaruskaya Navuka, Minsk. Pp. 351-543 (in Russian).

Sosnovska, S., \& Danylyk, I. (2017). Population structure of Carex dioica (Cyperaceae) in Ukraine under different growth conditions. Biodiversity: Research and Conservation, 46, 19-33.

Sosnovska, S., Danylyk, I., \& Serednytska, S. (2013). Distribution of the subgenus Psyllophora (Degl.) Peterm. (Carex L.) in Ukraine. Biodiversity: Research and Conservation, 29, 35-42.

Takhtajan, A. (2009). Flowering plants. 2nd ed. Springer.
Tatanov, I. V. (2004). Systema roda Bolboschoenus (Aschers.) Palla (Cyperaceae) [System of the genus Bolboschoenus (Aschers.) Palla (Cyperaceae)]. Novosti Sistematiki Vysshikh Rastenii, 36, 80-95 (in Russian).

Verloove, F. (2014). A conspectus of Cyperus s.l. (Cyperaceae) in Europe (incl. Azores, Madeira and Canary Islands), with emphasis on non-native naturalized species. Webbia: Journal of Plant Taxonomy and Geography, 69(2), 179-223.

Verloove, F., Mesterházy, A., \& Browning, J. (2016). Studies in Schoenoplectiella (Cyperaceae) in tropical West Africa. Phytotaxa, 283(1), 96-100.

Záveská Drábková, L. (2013). A survey of karyological phenomena in the Juncaceae with emphasis on chromosome number variation and evolution. Botanical Review, 79, 401-446.

Záveská Drábková, L., \& Kirschner, J. (2013). Oreojuncus, a new genus in the Juncaceae. Preslia, 85, 483-503.

Záveská Drábková, L., \& Vlček, C. (2010). Molecular phylogeny of the genus Luzula DC. (Juncaceae, Monocotyledones) based on plastome and nuclear ribosomal regions: A case of incongruence, incomplete lineage sorting and hybridisation. Molecular Phylogenetics and Evolution, 57(2), 536-551. 